\title{
Maternal high-fat diet during lactation reprograms the dopaminergic circuitry in mice
}

\author{
R.N. Lippert, ${ }^{1,2}$ S. Hess, ${ }^{3,4}$ P. Klemm, ${ }^{1}$ L.M. Burgeno, ${ }^{5}$ T. Jahans-Price, ${ }^{5}$ M.E. Walton, ${ }^{5}$ P. Kloppenburg, ${ }^{3,4}$ and J.C. Brüning ${ }^{1,2,4,6}$ \\ ${ }^{1}$ Department of Neuronal Control of Metabolism, Max Planck Institute for Metabolism Research, Cologne, Germany. ${ }^{2}$ National Center for Diabetes Research (DzD), Neuherberg, Cermany. ${ }^{3}$ Biocenter, Institute \\ for Zoology, and ${ }^{4}$ Cologne Excellence Cluster on Cellular Stress Responses in Aging-Associated Diseases (CECAD), University of Cologne, Cologne, Germany. ${ }^{5}$ Department of Experimental Psychology, \\ University of Oxford, Oxford, United Kingdom. ${ }^{6}$ Center for Endocrinology, Diabetes and Preventive Medicine (CEPD), University Hospital of Cologne, Cologne, Germany.
}

\begin{abstract}
The maternal perinatal environment modulates brain formation, and altered maternal nutrition has been linked to the development of metabolic and psychiatric disorders in the offspring. Here, we showed that maternal high-fat diet (HFD) feeding during lactation in mice elicits long-lasting changes in gene expression in the offspring's dopaminergic circuitry. This translated into silencing of dopaminergic midbrain neurons, reduced connectivity to their downstream targets, and reduced stimulus-evoked dopamine (DA) release in the striatum. Despite the attenuated activity of DA midbrain neurons, offspring from mothers exposed to HFD feeding exhibited a sexually dimorphic expression of DA-related phenotypes, i.e., hyperlocomotion in males and increased intake of palatable food and sucrose in females. These phenotypes arose from concomitantly increased spontaneous activity of D1 medium spiny neurons (MSNs) and profoundly decreased D2 MSN projections. Overall, we have unraveled a fundamental restructuring of dopaminergic circuitries upon time-restricted altered maternal nutrition to induce persistent behavioral changes in the offspring.
\end{abstract}

\section{Introduction}

Globally, rates of obesity and overweight far surpass underweight, with the prevalence of obesity in females exceeding that of males in all adult age categories (1). The etiology of obesity and associated metabolic disorders is multifold, including contributions of genetic, epigenetic, and environmental factors. Recent discoveries implicate metabolic perturbations in the maternal environment and during the perinatal period in the development of not only metabolic but also numerous other diseases, including neuropsychiatric disorders, such as attention-deficit/hyperactivity disorder (ADHD), autism, and cognitive impairment (2-5). Of increasing relevance is that rates of maternal obesity and overnutrition have continued to rise at alarming rates (>20\% in some Western cultures; ref. 6). Furthermore, independently of prepregnancy BMI status, $47 \%$ of women in Western cultures, from over 1 million cases studied, gained significantly more than the recommended amount of body weight during pregnancy, while only $25 \%$ gained less weight than recommended (7). The negative impact that overnutrition during this critical developmental period of the offspring has on brain development is not well understood at the molecular level.

Proper brain formation and function are directly influenced by the maternal perinatal environment in both humans and in rodents. Alterations in certain aspects of maternal physiology, including

\section{Related Commentary: p. 3416}

Conflict of interest: The authors have declared that no conflict of interest exists. Copyright: (5) 2020, American Society for Clinical Investigation.

Submitted: October 21, 2019; Accepted: March 26, 2020; Published: June 8, 2020.

Reference information: / Clin Invest. 2020;130(7):3761-3776.

https://doi.org/10.1172/JCl134412. stress and nutrition, have been implicated as key players in negatively affecting offspring brain formation (8). Previous work in rodents has shown that maternal obesity and maternal high-fat diet (HFD) consumption are linked to changes to the critical metabolic circuitries in the brain, specifically within the hypothalamus, resulting in obesity and metabolic dysfunction (refs. 9, 10; reviewed in ref. 11). Here agouti-related peptide (AgRP) and proopiomelanocortinexpressing (POMC-expressing) neurons in the arcuate nucleus of the hypothalamus make up critical metabolism-regulatory neuron populations, which adapt a plethora of physiological responses to the energy state of the organism. Specifically, we showed in a previous study that feeding mice an HFD during lactation compromises projection formation of POMC neurons to its intrahypothalamic projection sites, thereby predisposing the offspring to obesity and alterations in glucose metabolism. Interestingly, mutations in genes interfering with proper projection formation of melanocortin neurons have also been recently linked to rare cases of monogenic obesity in humans (12). Collectively, these findings highlight the notion that the same pathways deregulated as a consequence of altered maternal metabolism at the same time serve as the target for mutations in rare genetic disease.

However, in humans, many of the correlative deficits in behavior in children of mothers exposed to metabolic disturbances during pregnancy (i.e., as seen in ADHD and cognitive impairment) are closely linked to the dysfunctionality of dopaminergic neurocircuits $(4,13-15)$. Previous work has shown an effect of maternal prepregnancy obesity on aspects of dopamine (DA) system function and gene expression (16-19), but how specifically maternal gestational weight gain affects DA circuits via studies in rodent models as well as the global disturbances to brain structure, function, and overall behavior has not been characterized. 

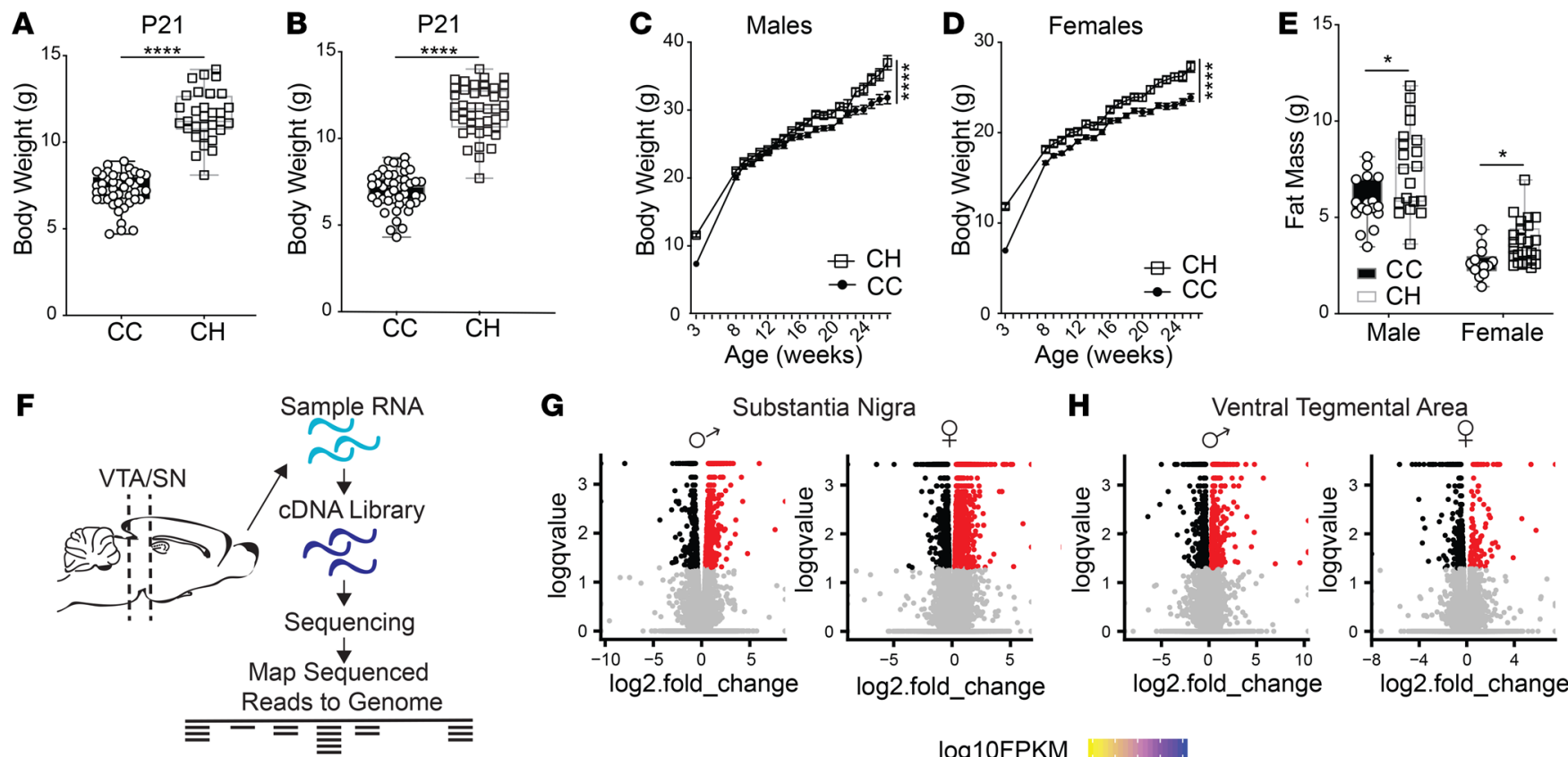

I

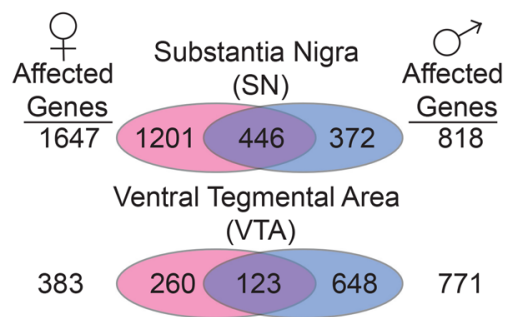

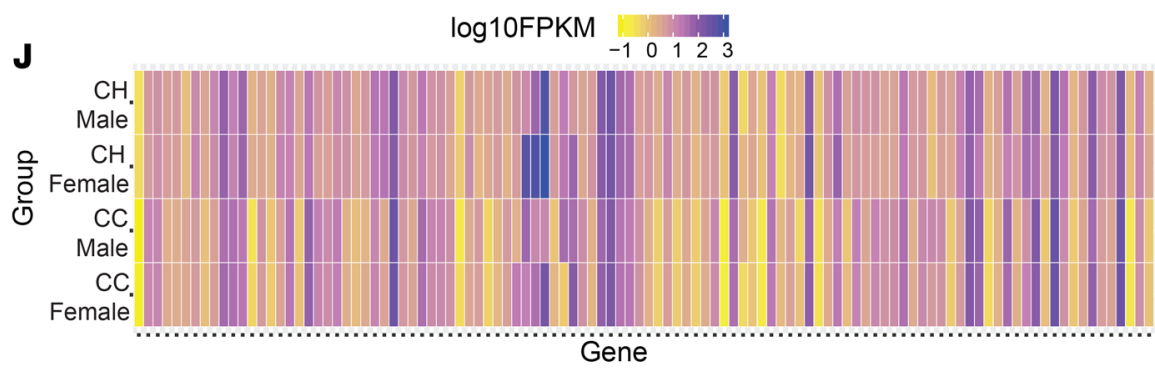

$\mathbf{K}$

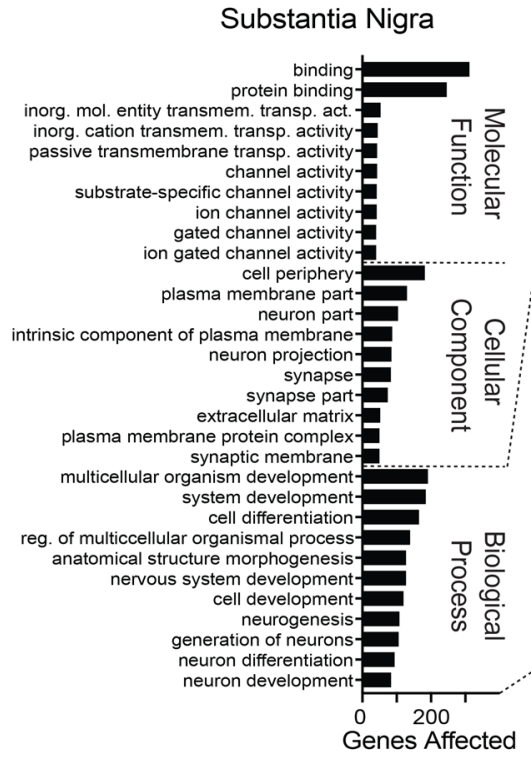

$\mathbf{L}$

Figure 1. Maternal HFD alters metabolic profile and causes changes to dopaminergic circuitry. (A and B) Body weight at P21 in (A) male control diet-exposed (CC, $n=49)$ and HFD-exposed mice during lactation ( $C H, n=31)$ and (B) female CC $(n=47)$ and CH $(n=43)$ offspring with or without HFD exposure during the lactation period. (C and D) Body weight in (C) CC versus CH males and (D) females through adulthood ( $n=10-34 /$ diet/sex/genotype/time point). (E) Body fat in CC versus CH males and females. (F) Strategy for midbrain dissection of VTA and SN tissue and subsequent RNA-sequencing pipeline ( $n=5-6$ mice per diet/ sex/brain region). (C) Volcano plots depicting significantly regulated genes in the male SN and female SN. (H) Volcano plots depicting significantly regulated genes in the male VTA and female VTA. Significance after correction for multiple testing plotted as $\log _{10}$ of the $Q$ value versus fold change over CC control group (full description of analysis pipeline in Methods section). (I) Significantly affected genes in males and females and overlapping genes. (J) Heatmap of top 100 overlapping genes shows high similarity between sexes of the same diet groups and dissimilarities between diet groups. (K) GO analysis of top hits in molecular function, cellular component, and biological function. (L) Tree map clustering GO terms based on common themes; square size indicates the absolute log of the $P$ value (larger $=$ more significant). ${ }^{*} P<0.05$, and ${ }^{* * *} P<0.0001,2$-sided Student's $t$ test (A, B, and $\mathbf{E}$ [female]), Welch's $t$ test (E [male], due to differences in variances), or mixed-effects analysis with repeated measures ( $\mathbf{C}$ and $\mathbf{D}$ ) and also with Bonferroni's post hoc analysis. 
In the present study, we employed a time-restricted dietary manipulation of mice during lactation, as this, from a neurodevelopmental aspect, most closely correlates to the processes affecting the third trimester of pregnancy in humans, such as the continued neurogenesis, increase in synaptogenesis, and drastic uptick in myelination within limbic structures that occur during this period in both rodents and humans (20-24). Previously, it was shown that this developmental time period is the most affected by maternal diet with regard to metabolic outcomes and hypothalamic function in the offspring $(9,25)$, but it had not been studied in the context of dopaminergic function. Furthermore, studies in humans have shown that elevated gestational weight gain alone, independently of prepregnancy weight status, is sufficient to drive the risk of developmental disorders (13-15). Therefore, it is critically important to understand the role of the maternal diet and altered maternal metabolism in this period of brain formation. We show that maternal HFD exposure during lactation is sufficient to elicit distinct changes in gene expression in key dopaminergic circuits of the offspring, specifically genes affecting neuronal development, ion channel expression, and locomotor behavior. Functionally, this translates into disturbed firing and silencing in a large proportion of DA midbrain neurons, reduced functional connectivity to their downstream target sites, and reduced stimulus-evoked DA release in the striatum. Strikingly, despite reduced DA neuron activity, their reduced connectivity, and decreased DA release in their target sites, offspring from mothers exposed to HFD feeding during lactation exhibited elevation in DA-related behaviors, namely hyperlocomotion in males and increased intake of palatable food and sucrose in females. These phenotypes result from concomitant direct changes in downstream medium spiny neurons (MSNs). Here, D1 MSNs increase rates of spontaneous activity and response to a stimulus in the presence of decreased D2 MSN projections. Overall, we have uncovered a fundamental and permanent restructuring of basal ganglia circuitry in response to HFD insult during early postnatal development.

\section{Results}

Maternal HFD permanently reprograms the $m R N A$ profile of midbrain $D A$ neurons. In order to study the effect of maternal diet on brain development, we used a previously developed maternal dietary manipulation paradigm to expose WT animals to an HFD during the lactation period (9). Briefly, the mothers were maintained on a standardized control diet from 4 weeks of age and were paired with males at 11 weeks of age; upon birth of the litter, mothers were switched to HFD or maintained on the control diet for the duration of the lactation period. At P21, all offspring were weaned onto the control diet for the duration of the experiment, ensuring that the dietary manipulation occurred only during the lactation period. Using this model, we showed an increased body weight in offspring of mothers receiving HFD during lactation (referred to as $\mathrm{CH}$ ) versus those only receiving control diet (referred to as CC; Figure 1, A and B) as well as throughout the life span (Figure 1, C and D). This increased body weight was in part due to an accumulation of body fat in both males and females (Figure 1E).

After establishing that the modified model successfully recapitulated the previously reported metabolic phenotype associated with maternal HFD exposure during lactation, we moved into specifically focusing on potential changes in the dopaminergic system of these animals. To do this, the dopaminergic midbrain, specifically the ventral tegmental area (VTA) and substantia nigra $(\mathrm{SN})$, were dissected from adult offspring (6 months of age) and subjected to mRNA-sequencing (Figure $1 \mathrm{~F}$ and Supplemental Methods; supplemental material available online with this article; https://doi.org/10.1172/JCI134412DS1). After correction for multiple testing, a robust number of genes remained changed in adulthood in offspring of mothers receiving HFD exposure during lactation. Specifically, in male CC versus $\mathrm{CH}$ animals, 818 and 711 genes were significantly altered in the SN and VTA, respectively (Figure 1, G-I). In females, 1647 and 383 genes were significantly affected in SN and VTA tissue (Figure 1, G-I). In the SN, 446 affected genes were found to overlap between sexes, with 123 overlapping in the VTA (Figure 1I). Analysis of the top 100 most significant overlapping genes in this region showed high similarity within sex and dissimilarity between maternal dietary manipulation groups (Figure 1J). Gene Ontology (GO) analysis and subsequent $\mathrm{GO}$ term clustering of the overlapping transcriptional changes uncovered the affected biological functions comprising in large part neuron development, ion transport, regulation of membrane potential, DA metabolism, locomotion, and behavior (Figure $1, \mathrm{~K}$ and $\mathrm{L}$ ). Additional GO term analysis and clustering of VTA samples displayed a distinct transcriptional profile highlighting ion transmembrane transport and chemical synaptic transmission but showing no enrichment of development-related genes (Supplemental Figure 1, A and B). Collectively, these data show that maternal HFD exposure during lactation fundamentally affects the midbrain transcriptome in the adult offspring.

Neuron development is altered in DA neurons and subsequent neuronal projections. As the most significantly affected cluster of genes in the SN samples pertain to neuron development, we focused on investigating the morphology of the SN dopaminergic neuron. Eight-week-old WT CC and CH offspring were analyzed via immunohistochemistry for DA neuron projections from the SN in both male and female animals. Using immunohistochemical analysis of tyrosine hydroxylase (TH), the rate-limiting enzyme in DA synthesis, as a marker of DA neurons, a significant reduction in the density of $\mathrm{TH}^{+}$signal within the $\mathrm{SN}$ pars reticulata (CC $1.000 \pm 0.1241$ vs. $\mathrm{CH} 0.3772 \pm 0.1186, P=0.0032$; Figure $2, \mathrm{~A}-\mathrm{C}$, and $\mathrm{G}$, and Supplemental Figure $2 \mathrm{C}$ ) as well as a reduction in the density of the labeled nigrostriatal tract neuronal fibers was noted (CC $1.000 \pm 0.1563$ vs. $\mathrm{CH} 0.4408 \pm 0.2359, P=0.0189$; Figure 2, D-F, and H, and Supplemental Figure 2C). Further analysis of the NuAcc core showed a significant reduction in $\mathrm{TH}$ staining in males and females, and analysis of the VTA showed no difference between diet groups (Supplemental Figure 2, A, B, and D). An additional assessment of the number of TH-, calbindin-, or Aldh1a1-labeled DA neuron cell bodies in unilateral sections of the VTA and SN showed no difference in total number of labeled neurons in various rostral to caudal regions of the dopaminergic midbrain (Supplemental Figure 2, E-G). Thus, maternal HFD feeding during lactation impairs DA neuron projection development without major alterations in DA neuron numbers.

Maternal HFD changes the firing properties of midbrain DA neurons. We next aimed to compare the firing properties of midbrain DA neurons in offspring of mothers exposed to CD or HFD during 
A

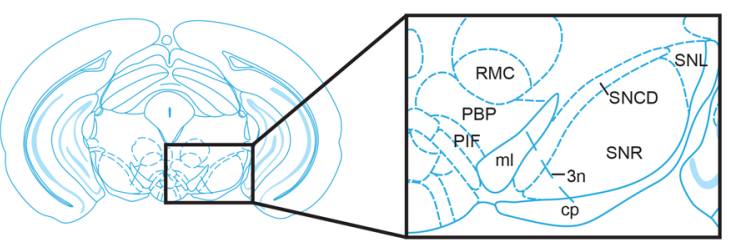

B

C

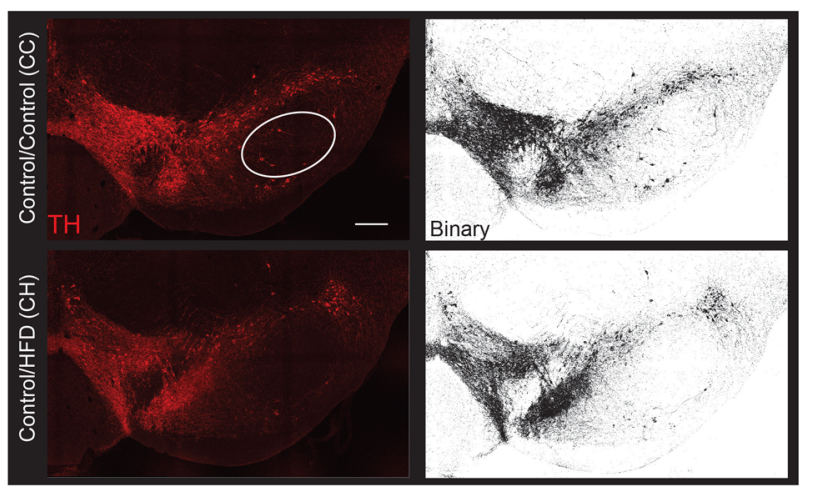

G

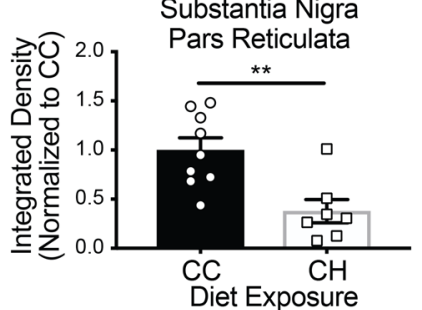

D

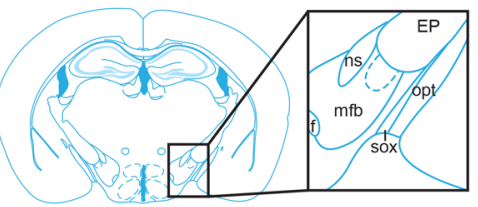

E
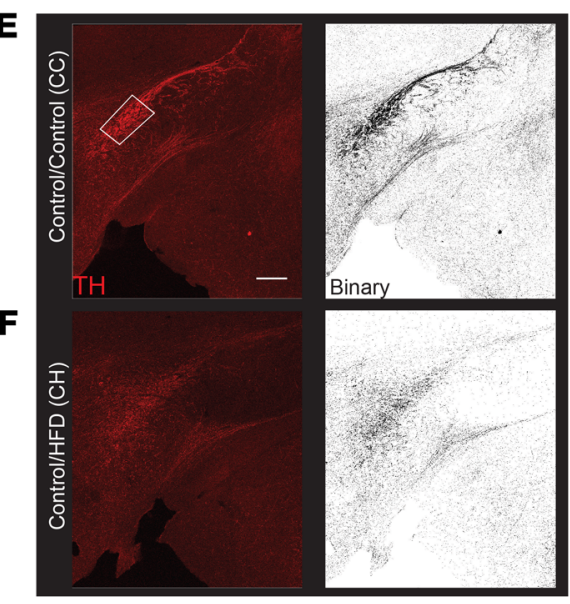

H

Nigrostriatal Tract

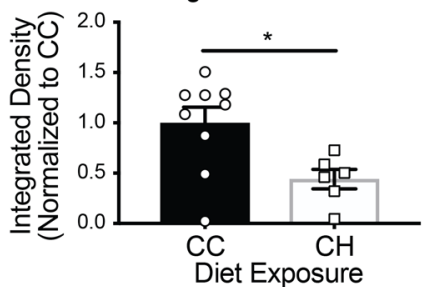

Figure 2. Maternal HFD negatively affects neuron development in the midbrain SN dopaminergic circuitry. (A) Reference for midbrain substructure anatomical location. Labeling of DA neurons in the SN and VTA using TH as a marker followed by binarization of the signal for analysis in (B) CC and (C) CH offspring. (D) Reference for nigrostriatal tract anatomical location. Additional IHC labeling in the nigrostriatal tract of (E) CC and (F) CH offspring. Quantification of the intensity of staining in the (G) SN pars reticulata (white oval in B) and corresponding (H) nigrostriatal tract (white box in E; $n=6-9$ per diet group). Scale bars: $500 \mu \mathrm{m}$. ${ }^{*} P<0.05$, and ${ }^{* *} P<0.01,2$-sided Student's $t$ test. RMC, red magnocellular nucleus; PBP, parabrachial pigmented nucleus; PIF, parainterfascicular nucleus; $\mathrm{ml}$, medial lemniscus; 3n, oculomotor nerve; $\mathrm{cp}$, cerebral peduncle; SNCD, substantia nigra, compact part; SNR, substantia nigra, reticular part; SNL, substantia nigra, lateral part; f, fornix; ns, nigrostriatal tract; mfb, medial forebrain bundle; EP, entopeduncular nucleus; opt, optic tract; sox, supraoptic decussation.

lactation. To this end, we performed perforated patch-clamp recordings from 14- to 17-week-old CC and CH offspring. DA neurons in the SN were identified by their characteristic electrophysiological phenotype (e.g., large "sag" potential upon hyperpolarization, broad action potentials [refs. 26-29 and Figure 3, A and B], and/or by their tdTomato fluorescence, as described in Methods). The firing patterns of DA neurons were classified into 3 categories: pacemaking, nonpacemaking, and silent (Figure 3B and Supplemental Figure 3, $\mathrm{H}$ and I; see Methods). In DA neurons of $\mathrm{CH}$ offspring, we found a smaller proportion of pacemaking neurons (Figure 3C; CC 64\% [22/34], CH 44\% [20/45]) and a significant larger proportion of silent neurons than in CC offspring (Figure 3, $\mathrm{C}$ and D, Supplemental Figure 3I, and Supplemental Table 1; CC $5.9 \%$ [2/34], CH 24.4\% [11/45], $P=0.0338,2$-sided Fisher exact test). In addition, the latency for the first action potential during a depolarizing current step was significantly shorter in DA neurons of $\mathrm{CH}$ offspring (Figure 3, E and F, Supplemental Figure 3J, and Supplemental Table 1; CC $0.21 \pm 0.026 \mathrm{~s}, n=11$, CH $0.15 \pm$ $0.032 \mathrm{~s}, n=33 ; P=0.02$, 2-tailed Mann-Whitney $U$ test). In line with this, we observed a tendency toward a lower spike threshold in $\mathrm{CH}$ offspring (Supplemental Figure 3D; CC $-43.6 \pm 0.93 \mathrm{mV}$, $n=11 ; \mathrm{CH}-46.7 \pm 0.71 \mathrm{mV}, n=28, P=0.08,2$-tailed Mann-Whitney $U$ test). All other parameters measured were not different between dietary groups (Supplemental Figure 3, A-C and E-G, and Supplemental Table 1). The differences noted were consistent across sexes, with a reduction in pacemaking neurons, increase in the percentage of silent neurons, and decreased latency to fire in both male and female SN neurons (Supplemental Figure 3, H-J). To further characterize the midbrain neurons, we performed similar analysis of lateral VTA (IVTA) neurons, which would constitute neurons signaling within the mesoaccumbal circuit. No change in basal firing properties of these neurons was noted (Supplemental Figure 4, A-H, and Supplemental Table 2). Interestingly however, upon increasing current injection, neurons from $\mathrm{CH}$ animals, both males and females, showed an increase in mean action potential frequency (Supplemental Figure 4, I-K, and Supplemental Table 2). Taken together, consistent with the transcriptomic analysis, these data show that maternal HFD exposure during lactation impairs the characteristic firing properties of a large number of DA neurons in adulthood. 
A

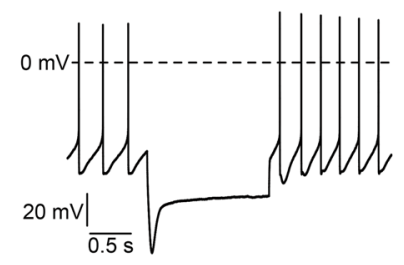

B

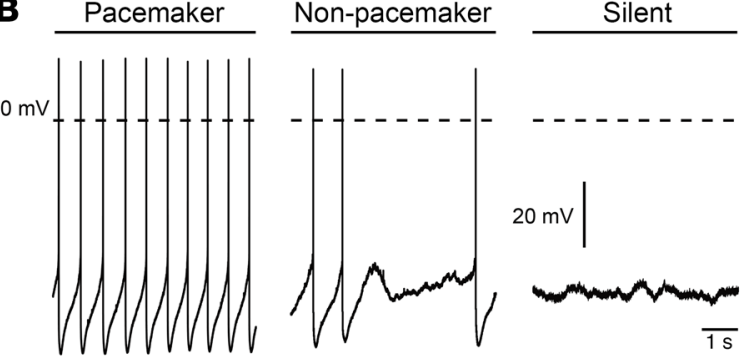

C

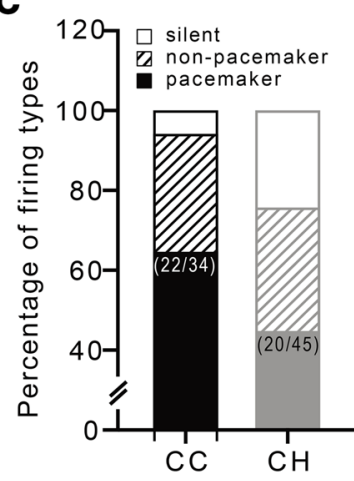

E

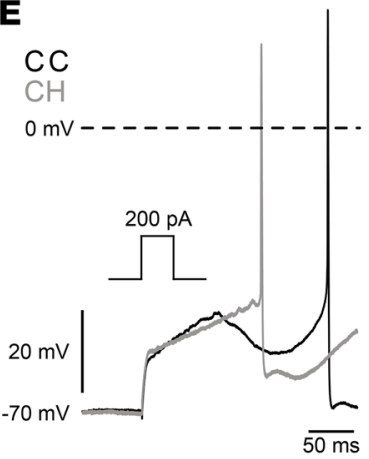

D

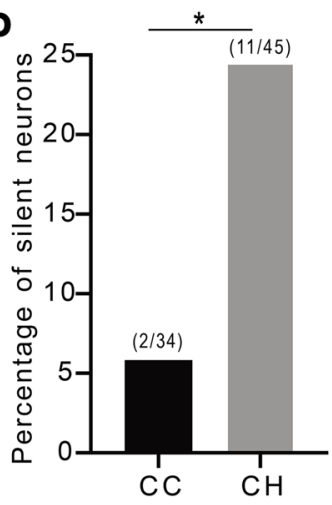

$\mathbf{F}$

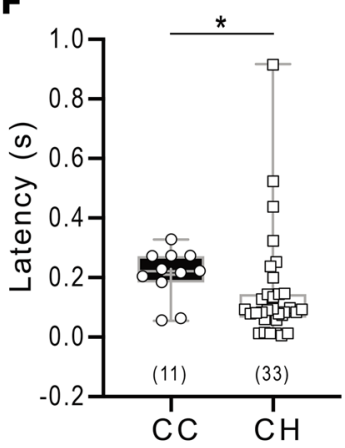

Figure 3. Electrophysiological properties of DA neurons in the SN are changed due to maternal HFD consumption. (A) Representative trace of DA neuron located in the SN pars compacta (left). Post hoc analysis of recorded neuron (right) shows colocalization of biocytin (recorded neuron; magenta) and the DAT (green). Scale bar: $20 \mu \mathrm{m}$. (B) Electrophysiological traces of defined DA neurons in the SN. (C) Distribution of DA neuronal firing types: pacemaking (solid), nonpacemaking (hatched), silent (white) neurons (CC [black] $n=34, \mathrm{CH}$ [gray] $n=45$ ). (D) Percentage ( $y$ axis) and total number (in parentheses) of silent neurons recorded. (E) Representative traces of latency to fire in CC and CH animals. (F) Quantification of latency to fire from all recorded neurons. ${ }^{*} P<0.05,2$-sided Fisher exact test (D) or 2-tailed Mann-Whitney $U$ test (F).

Evoked DA release in the ventral striatum is reduced in $\mathrm{CH}$ offspring. To study the consequences resulting from the observed alterations in firing properties of the midbrain DA neurons in the presence of decreased TH-labeled DA neuronal projections, we used fast scan cyclic voltammetry (FSCV) to assess evoked DA transients in the striatum in age-matched animals to the electrophysiological studies shown in Figure 3. We recently established a model of long-term monitoring of spontaneous and chemogenetically stimulated DA release specifically in the NuAcc core, which is one target region of IVTA neurons $(30,31)$. With the increased AP frequency upon current injection of IVTA neurons, we applied this FSCV stimulation protocol to the dietary manipulation model to analyze potential changes within the mesolimbic pathway (Figure 4, A and B). In CH animals, DA release in the NuAcc core, which was evoked by electrical stimulation of the midbrain, was reduced (highest stimulation parameter: CC $60.597 \pm 11.419 \mathrm{nA}$ vs. $\mathrm{CH} 24.053 \pm 5.769 \mathrm{nA}, P<0.0001$; Figure 4 , C-E). Post hoc analysis of the background current size, an indicator of electrode sensitivity (32), showed no differences between groups (Supplemental Figure 5). Collectively, these experiments support a decreased stimulus-evoked DA release in midbrain dopaminergic neuron target sites.

To further substantiate this notion, we employed a model of chemogenetic DA neuron stimulation. Here, we crossed mice allowing for Cre-dependent expression of the modified muscarinic receptor $\mathrm{hM} 3 \mathrm{D}_{\mathrm{Gq}}$ with those expressing the Cre recombinase in $\mathrm{DA}$ transporter-expressing (DAT-expressing) dopaminergic midbrain

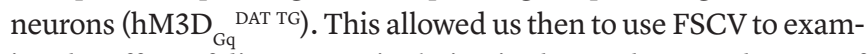
ine the effect of dietary manipulation in the mothers on the rate of spontaneous DA transients following chemogenetic stimulation. As shown previously, we found that chemogenetic activation of DA neurons upon treatment with clozapine- $n$-oxide $(\mathrm{CNO})$ promoted a clear increase in the rate of DA transients in CC animals, but not in $\mathrm{CH}$ animals (Figure 4, F and $\mathrm{G}$, total number DA transients $\mathrm{CC}_{\text {control }}$ $19.4 \pm 7.011, \mathrm{CH}_{\text {control }} 23.375 \pm 11.445, \mathrm{CC}\left[\mathrm{hM} 3 \mathrm{D}_{\mathrm{Gq}}{ }^{\mathrm{DAT}} \mathrm{TG}\right] 224.429$ $\pm 62.429, \mathrm{CH}\left[\mathrm{hM} 3 \mathrm{D}_{\mathrm{Gq}}^{\mathrm{DAT} T \mathrm{TG}}\right] 5.750 \pm 5.750, P_{\text {treatment }}=0.0272, P_{\text {diet }}$ $\left.=0.0129, P_{\text {interaction(diet x treatment) }}=0.0103\right)$. The data support that the altered firing properties and reduced TH labeling in projections of the DA midbrain neurons translate into a reduced capacity of DA neurons to release DA at downstream targets in the striatum.

Maternal HFD exposure results in increased locomotive behavior in male offspring. Given the drastic changes in the functional responses of DA neurons shown above, we assessed the resulting behavioral consequences in $\mathrm{CC}$ and $\mathrm{CH}$ animals with regard to DA-related behaviors. First, we investigated the well-characterized DA-associated features of locomotion in an open field test (OFT). Interestingly, here only males, and not females, displayed an enhanced locomotor response to novelty (Figure 5, A-D, and Supplemental Figure 6A). Specifically, significant increases in ambulatory episodes, jumping, rearing, and stereotypic behaviors 
A

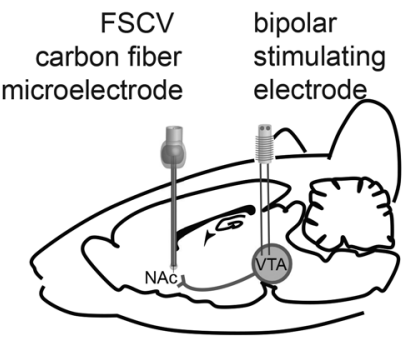

B

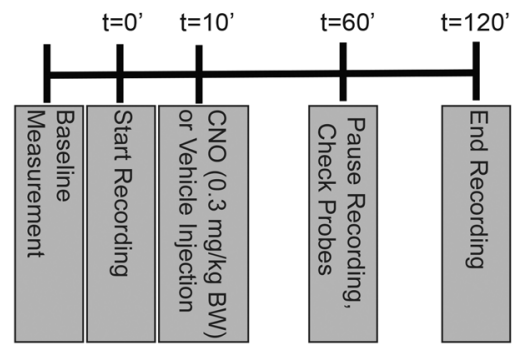

$\mathbf{C}$

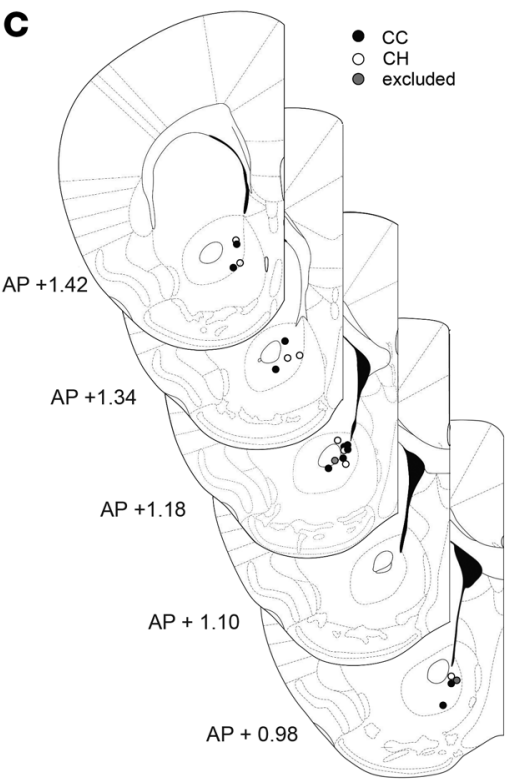

D
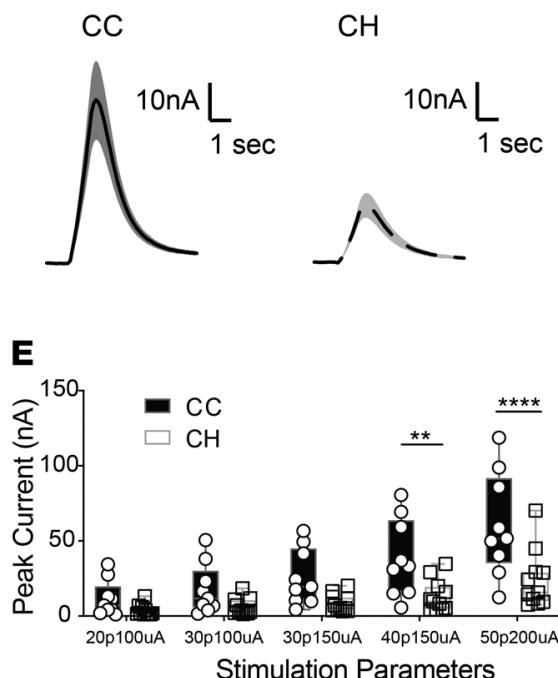

$\mathbf{F}$

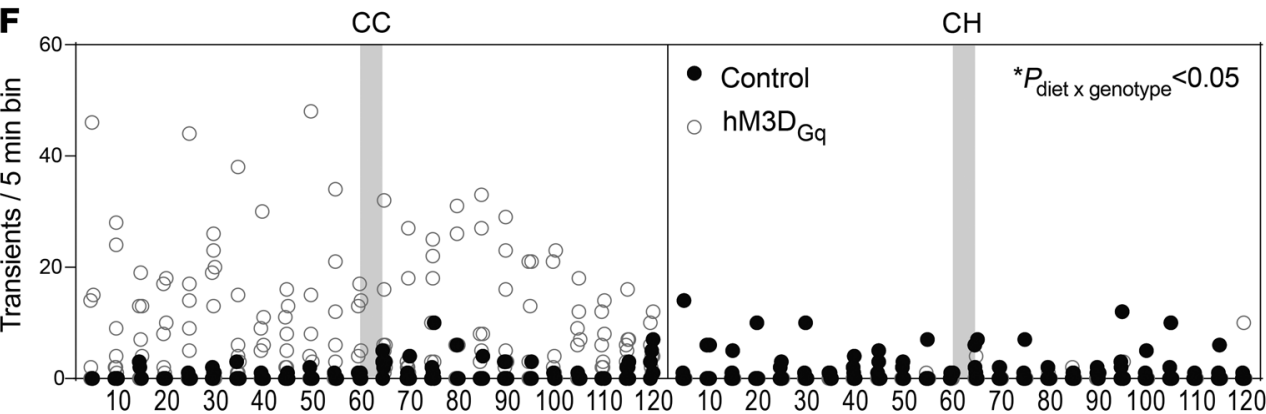

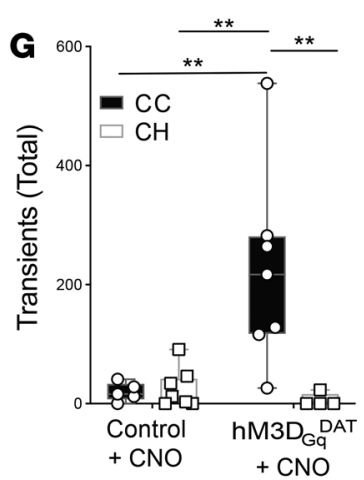

Figure 4. Reduced electrically and chemogenetically stimulated DA release in the ventral striatum detected with FSCV. (A) Representative schematic of placements for the stimulating and recording electrodes. (B) Experimental timeline. (C) Exact recording electrode placements determined by histological analysis. (D) Representative traces of the peak current detected in the CC (left) versus CH (right). (E) Peak current in CC (circles, $n=9$ ) versus CH (squares, $n=11$ ) animals in response to increasing electrical stimulation. $(\mathbf{F})$ Chemogenetically evoked DA transients in response to CNO application in the presence or absence of $\mathrm{hM}_{\mathrm{Cq}}$ expression. On the left are data from CC animals, and on the right are data from CH animals ( $n=4-8$ animals per diet/genotype). White circles depict CNO-stimulated transients detected over time. (G) Quantification of total DA transients detected throughout the duration of the study ( $n=4-8$ animals per diet/genotype). ${ }^{*} P<0.05,{ }^{* *} P<0.01$, and ${ }^{* * * *} P<0.0001,3$-way ANOVA (F) or 2-way ANOVA (E with repeated measures and $\left.\mathbf{G}\right)$.

were detected (Figure 5, E, F, I, and J). In females, only vertical behavior was significantly different, with no other alteration in ambulatory episodes, jumping, or stereotypic behaviors (Figure 5, $\mathrm{G}, \mathrm{H}, \mathrm{K}$, and L). This hyperlocomotive response in males was due to exposure to a novel environment, as repeated exposure to the chamber over subsequent days displayed no decipherable difference between dietary groups after day 1 (Figure 5, U and W). This behavior was also independent of an anxiety-related phenotype in the male $\mathrm{CH}$ animals, as proportional center distance traveled in the OFT throughout the test and time spent in the center during the first 5 minutes were unaltered between groups ( 5 minutes: $P=$ 0.9629; Supplemental Figure 6, B and C).

To probe whether the dysfunction of the dopaminergic circuitry had an earlier onset before alterations in normal behavior, we used hM3D ${ }_{G q}{ }^{\text {DAT TG }}$ and hM3D ${ }_{G q}{ }^{\text {DAT WT }}$ littermate controls with and without HFD exposure during lactation to assess DA-mediated behavior upon chemogenetic stimulation of DA neurons. At 2 months of age, all animals were acclimated to daily injections, subjected to the OFT immediately after receiving an intraperitoneal CNO injection, and tracked for 120 minutes. In all $\mathrm{Cre}^{-}$control groups, no increase in locomotor behavior was noted upon the application of CNO. In both CC and $\mathrm{CH}$ hM3D ${ }_{\mathrm{Gq}}{ }^{\mathrm{DAT}} \mathrm{TG}^{\mathrm{T}}$ animals, a significant increase in locomotion was seen in response to DA neuron stimulation, with vertical behavior and ambulatory episodes being increased (Figure 5, M, N, Q, and R). However, in $\mathrm{CH}$ $\mathrm{hM} 3 \mathrm{D}_{\mathrm{Gq}}{ }^{\mathrm{DAT}} \mathrm{TG}$ animals, a prolonged increase in locomotor-related behaviors was observed during the second hour following chemogenetic DA activation in comparison with $\mathrm{CC}$ hM3D ${ }_{\mathrm{Gq}}^{\mathrm{DAT}} \mathrm{TG}^{\mathrm{c}}$ controls (Figure 5, N and R). In females, while CNO treatment of CC and $\mathrm{CH} \mathrm{hM} 3 \mathrm{D}_{\mathrm{Gq}}{ }^{\mathrm{DAT}} \mathrm{TG}$ did elicit a slight but significant increase in locomotor-related behaviors, no differences due to HFD exposure were uncovered in both the initial phase and the second hour of behavioral assessment (Figure 5, O, P, S, and T).

As a further test of both novelty-induced locomotion and increased induction of DA-related signaling in HFD-exposed offspring, we performed the elevated zero maze (EZM) after application 
of CNO. An overall increase due to both dietary exposure and $\mathrm{hM} 3 \mathrm{D}_{\mathrm{G}}$ expression in males was noted $\left(P_{\text {genotype }}=0.0023 ; P_{\text {diet }}=0.0009 ;\right.$ Fig-

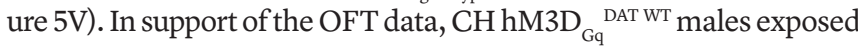
to the novel EZM apparatus showed a significant increase in locomotor movement compared with CChM3D ${ }_{\mathrm{Gq}}{ }^{\mathrm{DAT}} \mathrm{WT}^{\mathrm{T}}$ controls $(P=0.0204$; Figure 5V). Furthermore, upon application of CNO, CH DAT ${ }^{\mathrm{hM} 3 \mathrm{D}}$ animals significantly increased locomotor movement compared with CC hM3D ${ }_{\mathrm{Gq}}^{\text {DAT TG }}$ controls $(P=0.0413$; Figure 5V). In females, while the presence of the $\mathrm{hM} 3 \mathrm{D}_{\mathrm{Gq}}$ receptor did lead to an overall increase in locomotor movement $\left(P_{\text {genotype }}=0.0003\right)$, no effect was shown due to the diet $\left(P_{\text {diet }}=0.2132\right.$; Figure $\left.5 \mathrm{X}\right)$. These animals did not display any alteration in time spent in the open or closed arms of the EZM, further supporting the sexual dimorphism of the behavior as well as no obvious anxiety-related phenotype (Supplemental Figure 6, D-G). Collectively, despite a distinct reduction in DA-neuron firing, DA projections, and both electrically and chemogenetically evoked DA release, male $\mathrm{CH}$ offspring exhibit clear evidence of increased DA-dependent locomotion.

Consummatory behavior is altered in female $\mathrm{CH}$ offspring. The dopaminergic circuitry not only controls locomotor responses but also critically regulates behavior related to consumption of sucrose and palatable food. Thus, we first assessed responses to a sucrose solution in $\mathrm{CC}$ and $\mathrm{CH}$ offspring. At baseline, $\mathrm{CH}$ animals showed normal levels of water consumption that did not differ from those of CC counterparts (Figure 6, A and C). When given free access to both a $4 \%$ sucrose solution and water, all animals showed an increase over baseline of fluid consumption (Figure 6 , B, D, E, and G). All animals increased their total consumption of fluid, specifically increasing sucrose intake (Figure 6, E-H). In contrast to the locomotor phenotypes described above, however, $\mathrm{CH}$ females consumed significantly more fluid, driven by a significant increase in sucrose solution as compared with CC controls $\left(\mathrm{CC}_{\text {water }} 2.008 \pm 0.578 \mathrm{~mL}, \mathrm{CH}_{\text {water }} 1.212 \pm 0.351, \mathrm{CC}_{\text {Sucrose }} 3.208 \pm\right.$ $0.933 \mathrm{~mL}, \mathrm{CH}_{\text {Sucrose }} 7.7521 .215 \mathrm{~mL}, P_{\text {Solution }}=0.0001, P_{\text {diet }}=0.0561$, $P_{\text {interaction (diet } \times \text { solution) }}=0.0072$, Figure 6, D and H). This increase in sucrose consumption reflected a difference in preference between the groups: high preferers in the $\mathrm{CH}$ group showed a significantly increased preference for the sucrose solution that was not seen in the $\mathrm{CH}$ male group (Figure 6, I-L).

Further assessment of reward-related consummatory behavior was performed by challenging WT CC and $\mathrm{CH}$ animals with an HFD in adulthood. Eight-week-old CC and $\mathrm{CH}$ animals were either maintained on the control diet or given ad libitum access to a $60 \%$ HFD. In males, HFD-fed animals in both the CC and $\mathrm{CH}$ groups showed significant increases in body weight, but no differences in body weight or body fat percentage were detected between the 2 groups (Figure 6, M and N). In contrast, $\mathrm{CH}$ females showed a profound increase in body weight and body fat percentage in comparison with CC HFD controls (Figure 6, O and $\mathrm{P})$. Collectively, while male $\mathrm{CH}$ offspring exhibit a clear increase in DA-evoked locomotor responses, female $\mathrm{CH}$ offspring exhibit increased sucrose consumption, increased sucrose preference, and an increased response to HFD feeding in adulthood.

Striatal targets of dopaminergic circuitry are altered by maternal HFD consumption. Given the dichotomy of overall decreased DA neuronal function, connectivity, and release, but relative increases in DA-mediated behaviors, we assessed potential changes occurring in receptive target regions of the dopaminergic midbrain. To this end, we first measured gene expression changes via mRNA-sequencing in the dorsal and ventral striatum where the D1 and D2 MSNs reside and receive input from the DA neurons of the midbrain. Given the sexually dimorphic phenotypes in behavior, we assessed genetic profiles that were either common or unique to male or female after correction for multiple testing. In males, a total of 320 genes in the dorsal striatum and 298 genes in the ventral striatum were significantly altered between CC and $\mathrm{CH}$ offspring (Figure 7, $\mathrm{A}-\mathrm{C}$ ). In contrast, 823 and 672 genes were found to be significantly changed in the female striatum between $\mathrm{CC}$ and $\mathrm{CH}$ offspring (Figure 7, A-C). Given that in females there was an approximate 4 -fold increase in the total number of genes affected (Figure 7, A-C), we analyzed the function of these regulated genes in each sex individually by performing a GO analysis. Further comparison of these GO terms between males and females resulted in a high similarity between sexes of the GO terms affected and percentages of genes in each data set involved (Figure 7D). Therefore, despite the relative difference in total genes affected, the resulting outcomes show similar effects proportionally at the level of the GO term. Assessing the biological function of these GO terms, the majority of terms are linked with neuronal signaling and development (Figure 7D).

Since the striatum comprises primarily D1 and D2 MSNs, we compared our genetic changes in the dorsal and ventral striatum to a publicly available data set of mRNAs specifically expressed in D1 and D2 MSNs (33). This analysis revealed that D1 MSN-specific transcripts were enriched in the differentially expressed genes from the striatal samples in both male and female $\mathrm{CH}$ compared with CC offspring as compared with D2 MSN markers (73/224 [33\%] vs. 17/102 [17\%]; Supplemental Methods). These results indicate a predominant change in gene expression of D1 MSNs in the $\mathrm{CH}$-exposed animals.

Excitability and connectivity of striatal neurons are deregulated in $\mathrm{CH}$ animals. Given the predominant transcriptional changes in D1 MSNs of $\mathrm{CH}$ offspring, we assessed the electrophysiological properties of D1 MSNs of striatum. To do this, we crossed mice expressing the Cre recombinase under control of the DRD1a promoter (DRD1a ${ }^{\text {Cre }}$ ) with mice allowing for Cre-dependent expression of TdTomato (Rosa26-TdTomato ${ }^{\mathrm{fl} / \mathrm{fl}}$ ), which were then exposed to the same dietary manipulation during lactation as that described above. This allowed for specific monitoring of D1R-expressing MSNs, which make up the direct pathway and are known to stimulate locomotion (Figure 8, A and B). In accordance with the analysis of overlapping GO terms (Figure 7D), in both males and females, we measured a significantly depolarized resting membrane potential of D1 MSNs of $\mathrm{CH}$ offspring (Figure 8, C and D, and Supplemental Table 3; CC -82.2 $\pm 2.95 \mathrm{mV}, n=$ 20; CH -68.9 $\pm 4.03, n=22 ; P=0.02$, 2-tailed Mann-Whitney $U$ test). In line with this, we found that a significant proportion of D1 MSNs of $\mathrm{CH}$ offspring were spontaneously active (Figure 8E; CC 5\% [1/20], CH 31.8\% [7/22]; $P=0.0471$, Fisher exact test). In addition, we measured a reduction of the single spike after hyperpolarization in D1 MSN of CH offspring (Supplemental Figure 7C and Supplemental Table 3; CC $16.3 \pm 1.23 \mathrm{mV}, n=13$; CH $13.2 \pm$ $0.72 \mathrm{mV}, n=16 ; P=0.03$, 2-tailed Mann-Whitney $U$ test). Interestingly, in support of the sexually dimorphic phenotypes with regard 


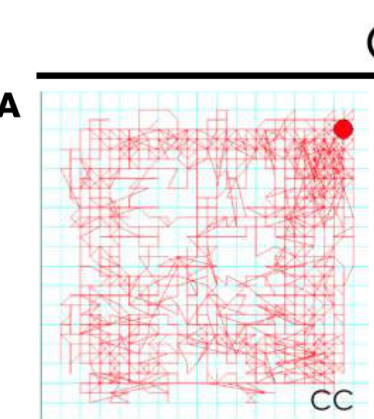

$0^{2}$
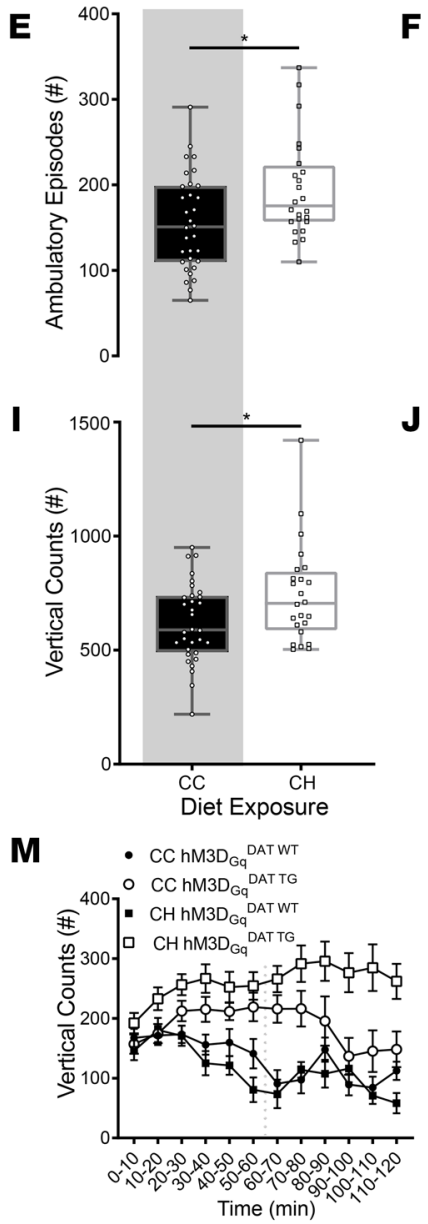

$\mathbf{Q}$
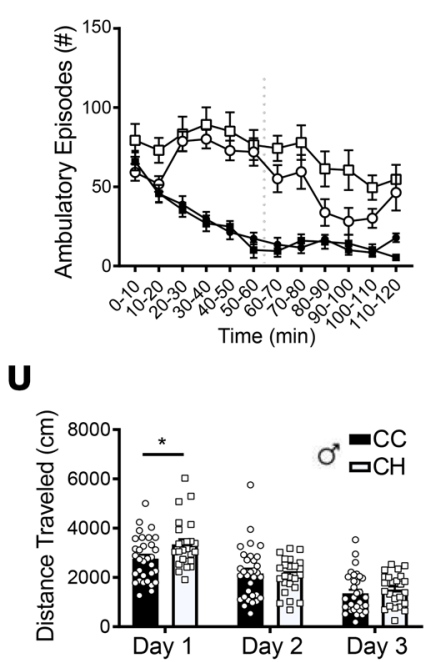

$\mathbf{F}$
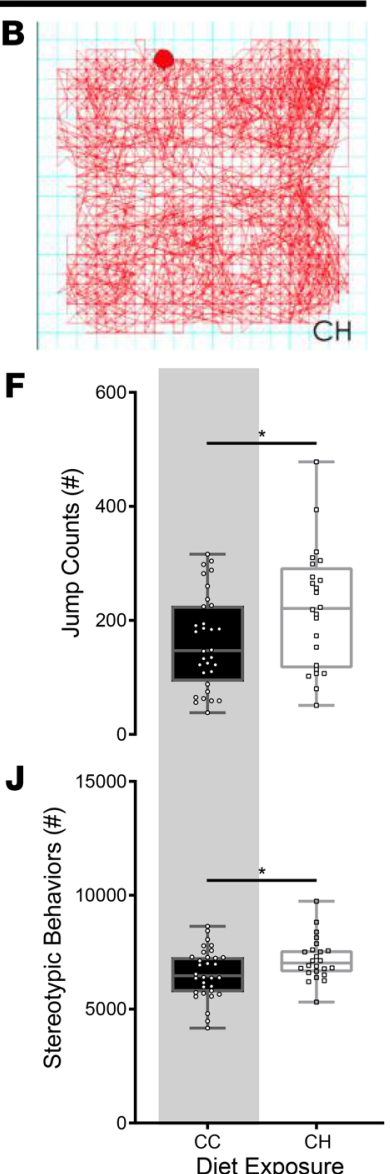

$\mathbf{N}$

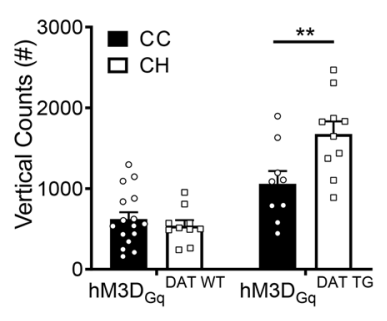

R

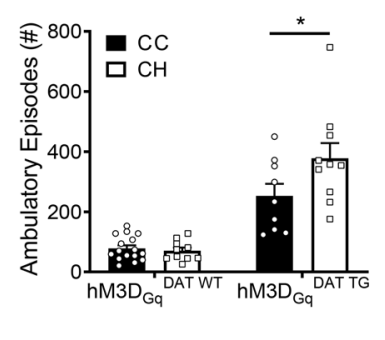

V

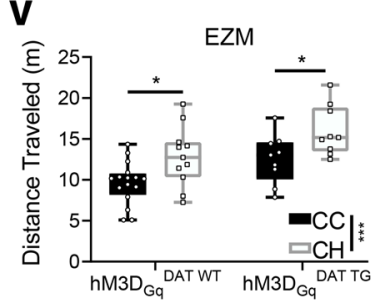

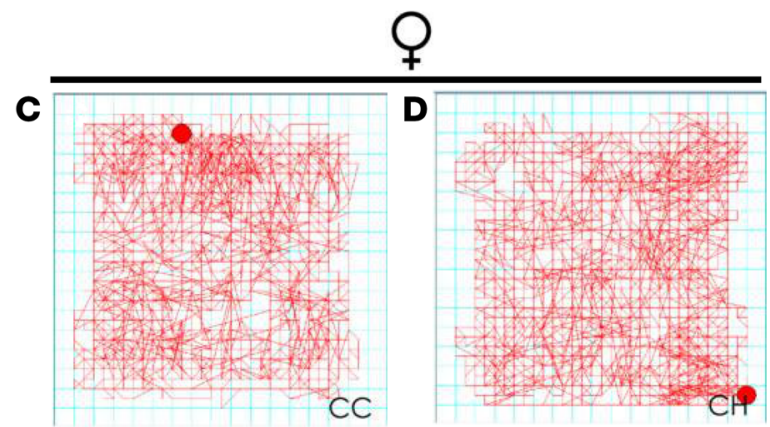

G

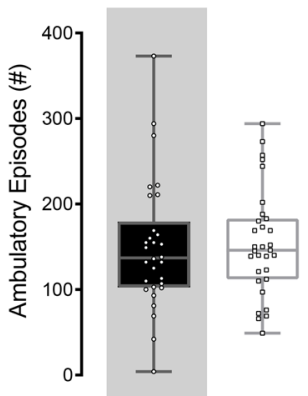

H

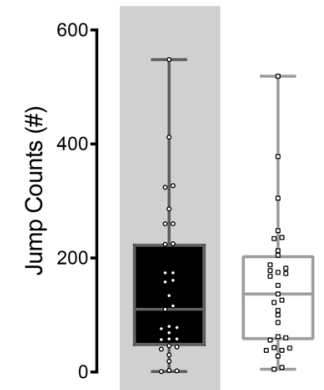

$\mathbf{K}$

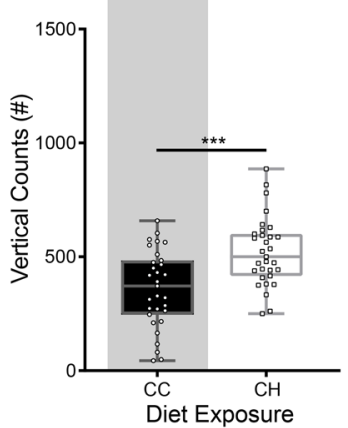

$\mathbf{L}$

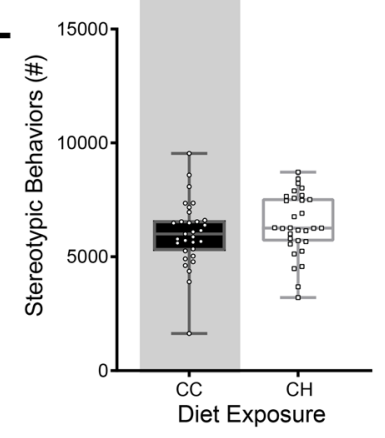

o
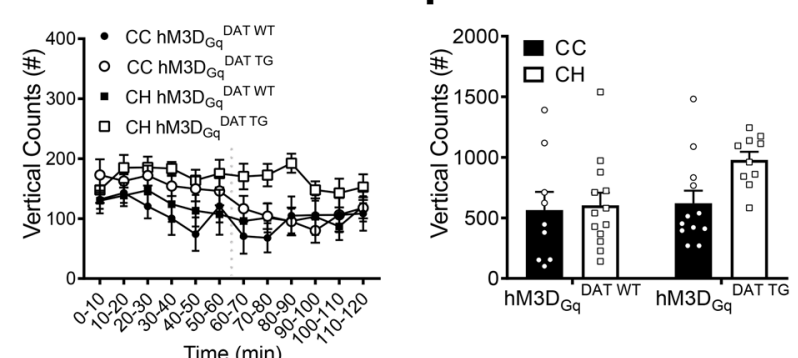

S
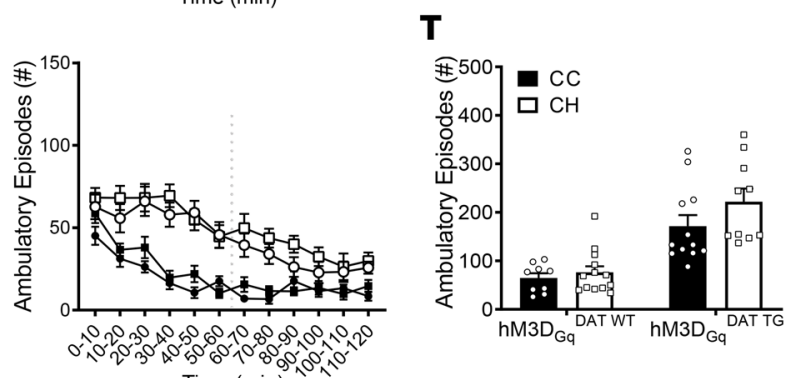

W
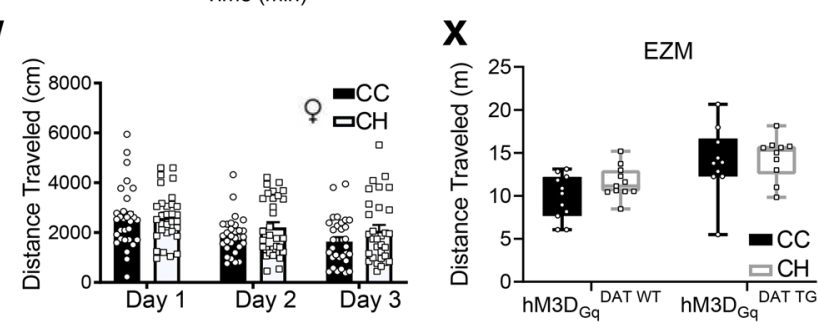
Figure 5. CH male offspring, and not females, exhibit hyperlocomotive behavior. (A-D) Representative tracking of animals in the OFT in a (A) CC male $(n=32)$, (B) CH male ( $n=24)$, (C) CC female $(n=31)$, and (D) CH female $(n=31)$. Quantification of ambulatory episodes $(\mathbf{E})$, jump counts $(\mathbf{F})$, rearing events (I), and stereotypic behaviors (J) in CC (circles) versus $\mathrm{CH}$ (squares) males at 6 months of age. Quantification of ambulatory episodes (G), jump counts (H), rearing events (K), and stereotypic behaviors (L) in CC (circles) versus CH (squares) females at 6 months of age. (M-T) Chemogenetic activation of young animals at 8 to 10 weeks of age tracked over time after CNO injection at $t=0^{\prime}$ followed by AUC analysis of the second hour ( $n=9-15$ per diet/genotype/sex, combination of at least 2 different experimental rounds). Vertical counts in males over time (M) and in the second hour (N). Vertical counts in females over time $(\mathbf{O})$ and in the second hour (P). Ambulatory episodes in males over time (Q) and in the second hour (R). Ambulatory episodes in females over time (S) and in the second hour $(\mathbf{T})$. Locomotive behavior in 6 -month-old $\mathrm{CC}$ versus $\mathrm{CH}$ males (U) and females (W) with repeated daily exposure to the OFT. EZM total distance traveled in male $(\mathbf{V})$ and female $(\mathbf{X})$ animals $(n=7-15$ per diet/sex/ genotype; results indicate a combination of at least 2 separate experiments). ${ }^{*} P<0.05,{ }^{* *} P<0.01$, and ${ }^{* * *} P<0.001$, Student's $t$ test $(\mathbf{E}-\mathbf{L})$ as well as a mixed effects analysis ( $\mathbf{M}, \mathbf{O}, \mathbf{Q}$, and $\mathbf{S}$ ) or 2-way ANOVA (N, $\mathbf{P}, \mathbf{R}$, $\mathbf{T}, \mathbf{V}, \mathbf{X}, \mathbf{U}$, and $\mathbf{W}$ with repeated measures).

to locomotor behavior, in males, we detected a selective increase in excitability as measured by a series of depolarizing current steps in D1 MSNs in the dorsal striatum of $\mathrm{CH}$ compared with CC mice (Figure 8, F and G, and Supplemental Table 3; $P=0.0009$, F test). This finding was not seen in females (Figure $8, \mathrm{H}$ and $\mathrm{I} ; P=0.9323$, F test). Analysis of the ventral striatal NuAcc neurons showed no difference in any parameter measured (Supplemental Figure 7 and Supplemental Table 4). Thus, the increased excitability of D1 MSNs in dorsal striatum of male $\mathrm{CH}$ offspring is consistent with their increased locomotion in response to novelty.

Finally, we aimed at investigating the downstream circuitry wiring of D1 and D2 MSNs in CC and CH offspring. Thus, to visualize projections of D2 MSNs and to complement the models where D1 MSNs are visualized via Tomato expression (DRD1a ${ }^{\text {TOM }}$ ), we crossed R26-TdTomato ${ }^{\mathrm{A} / \mathrm{l}}$ mice with mice that express the Cre recombinase under control of the Adora2a promoter specifically in postsynaptic D2 MSNs (Adora2a ${ }^{\mathrm{Cre}}$ ) resulting in Adora2 $\mathrm{a}^{\mathrm{TOM}}$ mice. Then we performed whole brain imaging of the neuronal connectivity of these 2 neuronal populations upon dietary manipulation of the mothers during lactation. We measured no detectable differences in signal intensity between DRD1a ${ }^{\text {TOM }} \mathrm{CC}$ and $\mathrm{CH}$ animals in the striatum, globus pallidus, and SN (Figure 8, J and $\mathrm{K}$ ). Strikingly, however, Adora $2 \mathrm{a}^{\text {TOM }} \mathrm{CH}$ animals displayed a robust decrease in neuronal projections in the globus pallidus and ventral pallidum (Figure 8, $\mathrm{L}$ and $\mathrm{M}$ ).

Previous studies in the field show that D1R activation, either pharmacologically or optogenetically, increases stereotypic behavior and overall locomotion (34-36). In order to assess whole animal behavioral changes as a response to the alteration in D1 MSN-firing properties, a cohort of $\mathrm{CH}$ males and females were exposed to the novel OFT chamber coupled with injection of a D1R agonist, SKF38393, or saline. In CH male animals that were naive to the OFT chamber, the novelty of the chamber environment led to an increase in stereotypic movements, as shown previously in Figure 5, and this was to the same magnitude as that shown by the animals receiving SKF38393 injection coupled with the novel environment (Supplemental Figure 8A). However, when animals were previously exposed to the chamber, in order to eliminate the novelty response, only the animals receiving SKF38393 showed significantly elevated stereotypic movement (repeated exposure: $\mathrm{CH}+\mathrm{SAL}$ vs. $\mathrm{CH}+\mathrm{SKF} 38393 P=0.0048 ; P_{\text {treatment }}=$ $0.0049, P_{\text {time }}=0.0434$; Supplemental Figure 8A). In females, this difference was not noted (Supplemental Figure 8B). Collectively, this decrease in D2 MSN innervation of the inhibitory indirect pathway in the presence of an increased excitability of D1 MSNs in the direct pathway may jointly operate to mediate the increased expression of DA-related behaviors in response to maternal diet manipulation during lactation despite an attenuated excitability and DA release of dopaminergic midbrain neurons.

\section{Discussion}

Altered maternal metabolism during pregnancy represents an important determinant for a plethora of diseases in the offspring including metabolic disorders, such as obesity, type 2 diabetes mellitus, and hypertension, and also an increased risk for neuropsychiatric disorders. While the molecular basis underlying this effect of "metabolic imprinting" has been widely investigated with respect to the role of epigenetic alterations of gene expression, an alternative model assigns alterations in neuronal development of key neurocircuits during critical periods of development a potential role in this process. We have previously demonstrated that feeding female mice an HFD selectively during lactation is sufficient to alter metabolic regulation in the offspring in the long term, predisposing them for obesity and altered glucose homeostasis (9). Mechanistically, we demonstrated that impairment of projection formation of key feeding regulatory POMC neurons during this period at least in part explains this phenomenon (9). Interestingly, it has been revealed recently that reduced POMC neuron expression of neuropilin-2 (the receptor for semaphorin-3) impairs POMC neuron projections similarly to what was observed in our dietary manipulation model (12). Mutations in semaphorin-3 and its signaling components result in obesity in zebrafish and mice as well as human carriers of semaphorin-3 mutations. Thus, altered neuronal projection formation during critical developmental periods provides a unifying mechanism for metabolic disorders occurring as a consequence of maternal programming as well as cases of rare monogenic obesity (12). Of note, projection formation, refinement, and maturation not only of melanocortin neurons but also of dopaminergic neurons in mice occurs during lactation, while in humans, it takes place during the last trimester of pregnancy (reviewed in refs. 37-39). Thus, altered neuronal development as a consequence of alterations in maternal metabolism likely has wider implications than those demonstrated for the key homeostatic hypothalamic melanocortin circuitry. Another candidate circuitry is represented here in dopaminergic midbrain neurons, which exhibit profound control over reward-associated feeding behavior largely viewed as a key regulator of hedonic feeding via mesolimbic circuitry in addition to the well-documented role of the nigrostriatal dopaminergic circuitry in the regulation of locomotor behavior (ref. 40; reviewed in refs. 41, 42). In accordance with this, obesity-associated alterations of dopaminergic neurons contribute to obesity development, and 


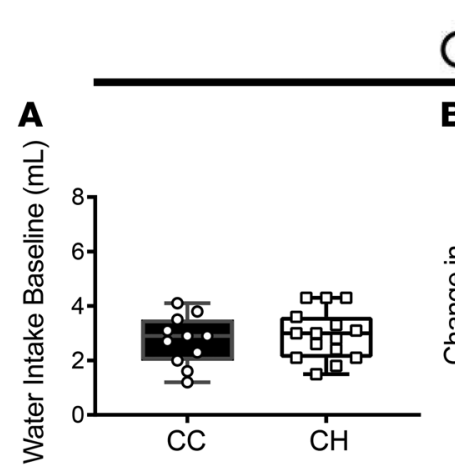

$0^{n}$
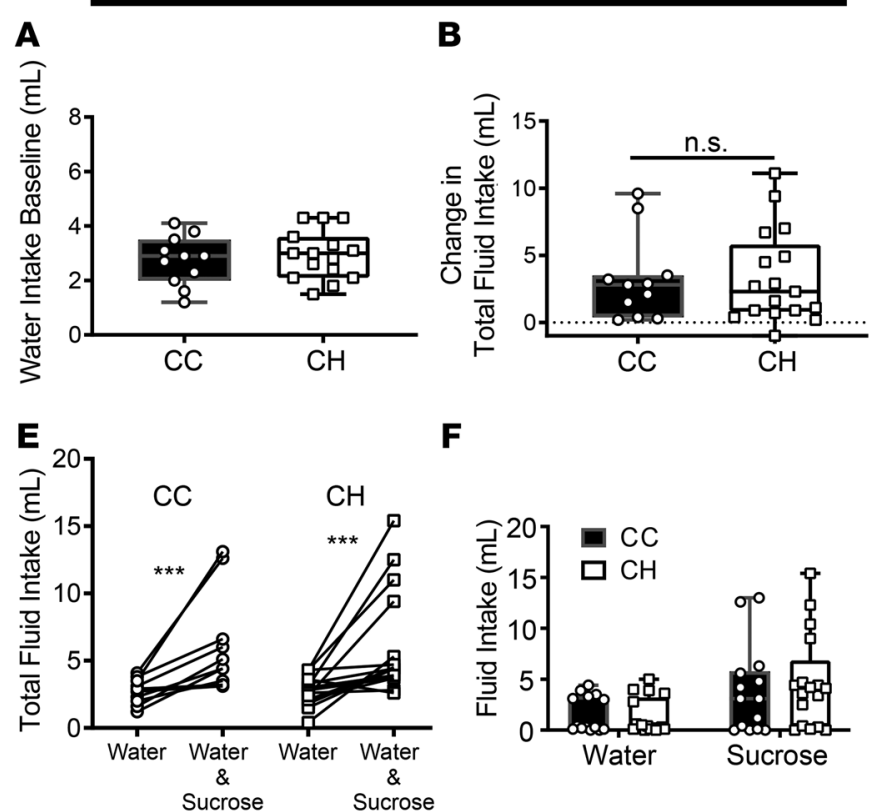

F

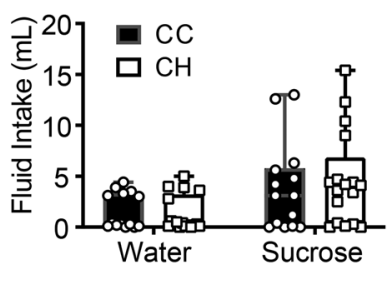

I

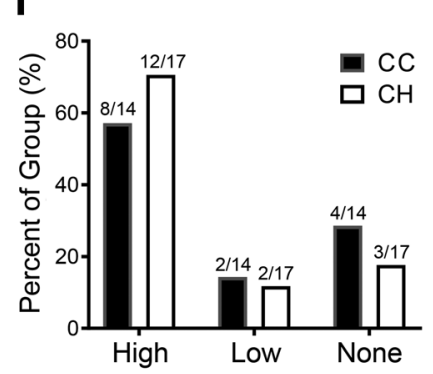

J

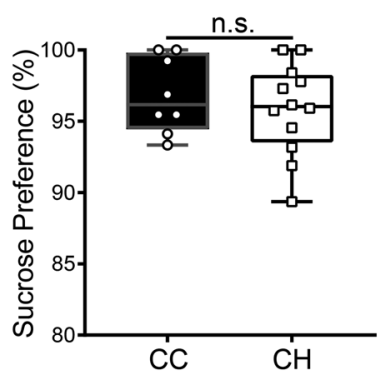

M

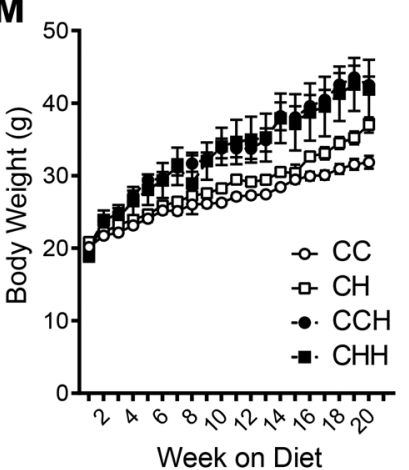

$\mathbf{N}$

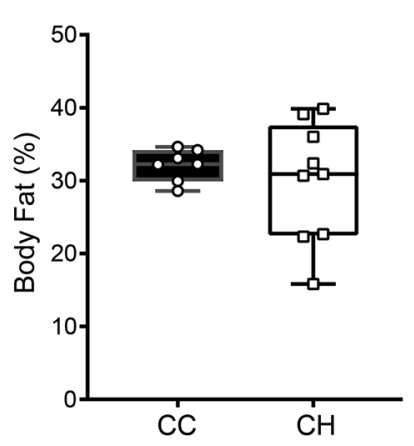

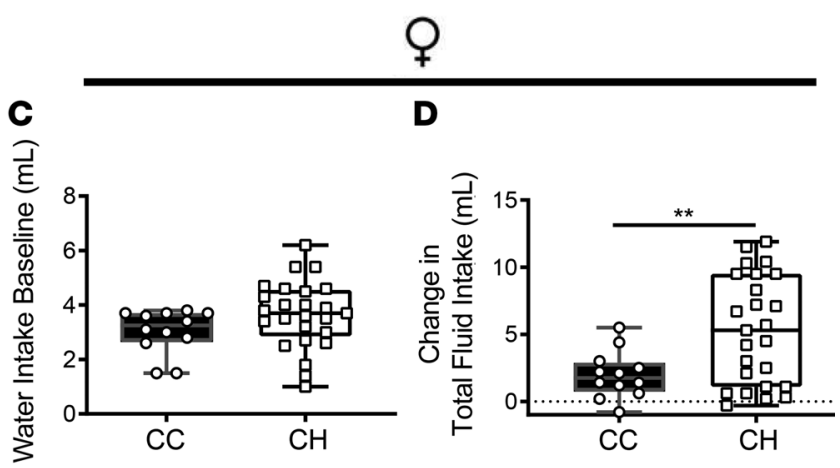

G

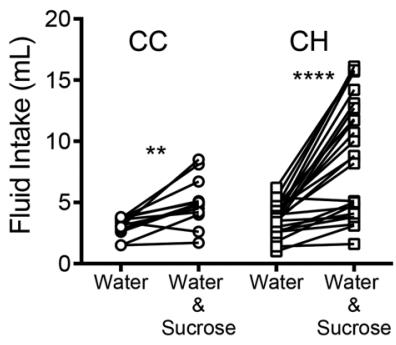

H
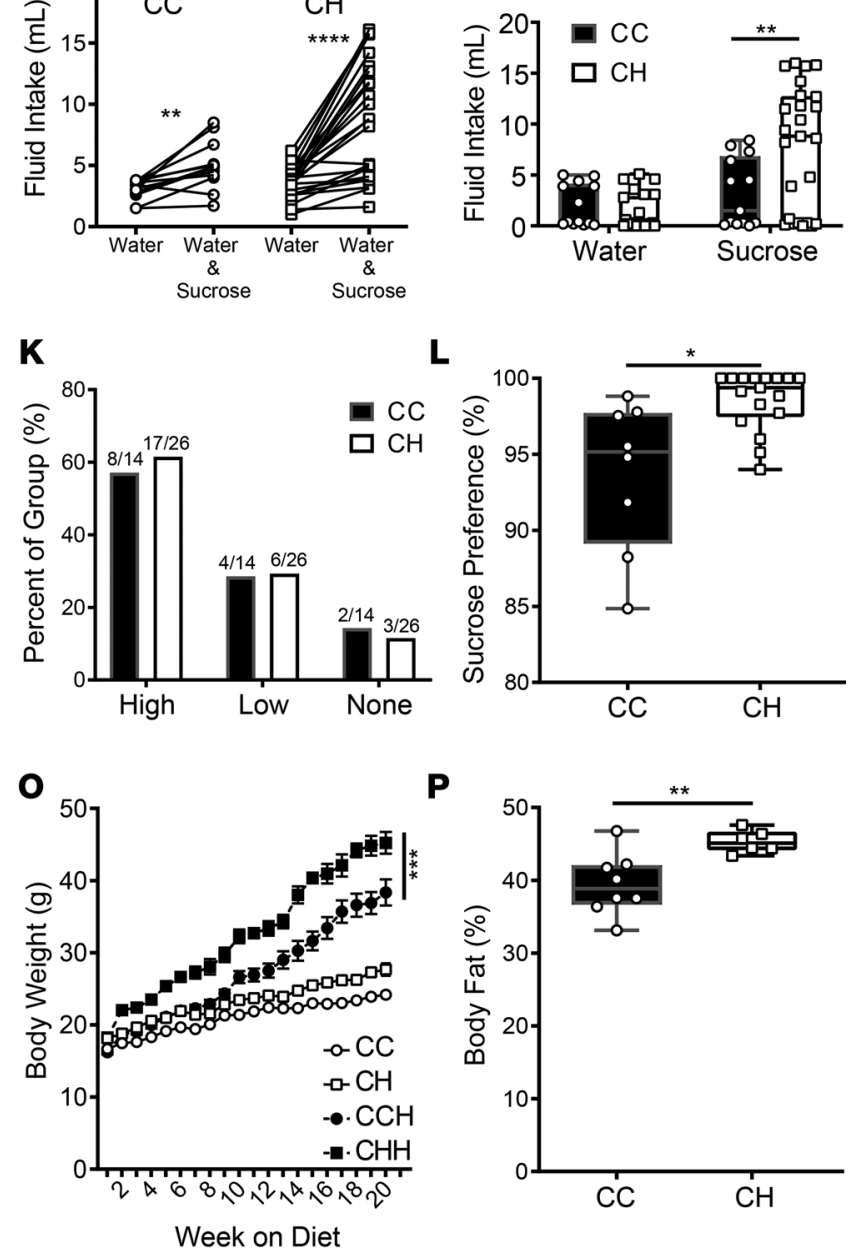

Figure 6. CH female offspring, and not males, exhibit increased consumption and body weight in response to palatable substances. Baseline water intake in CC and CH (A) males ( $n=11$ vs. 16) and (C) females ( $n=13$ vs. 25) shows no difference between genotypes. Change in fluid intake in the presence of $4 \%$ sucrose and water in CC and CH (E) males and (C) females. Total fluid intake of water and $4 \%$ sucrose solution combined in CC and CH (B) males and (D) females as compared with water baseline. Contribution of water intake and $4 \%$ sucrose intake in CC and CH (F) males and (H) females. Breakdown of preference for $4 \%$ sucrose into high (>80\%), low (>80\%), and no preference for sucrose groups in CC and CH (I) males and (K) females. Sucrose preference in CC and CH (J) males and (L) females in the high preferring group. Body weight gain on HFD or control diet in CC and CH (M) males ( $n=7-34$ per time point/diet) and (0) females ( $n=7-32$ per time point/diet). Body fat percentage on HFD in CC and CH (N) males $\left(n=7\right.$ vs. 9) and (P) females $\left(n=8\right.$ vs. 7). ${ }^{*} P<0.05 ;{ }^{* *} P<0.01 ;{ }^{* * *} P<0.001 ;{ }^{* * * *} P<0.0001$, 2-sided Student's $t$ test (A-E, G, J, L, and $\mathbf{N}-\mathbf{P})$, 2-way ANOVA with Bonferroni's post hoc analysis (F and $\mathbf{H}$ ), or mixed effects analysis (M and $\mathbf{0})$.

altered DA action has been identified in humans suffering from obesity and metabolic disorders (43-48). Moreover, dopaminergic midbrain neurons are likely effectors of signaling by the food intake-suppressing hormone leptin (49-52).
Here, our results provide clear evidence that maternal HFD feeding during the restricted, key developmental period of lactation has profound and long-lasting effects on the dopaminergic circuitry in offspring. Previous studies have proposed a link 
A

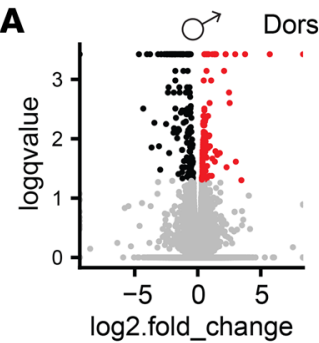

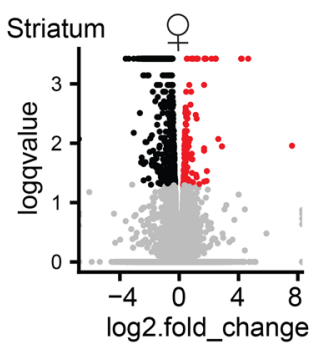

B

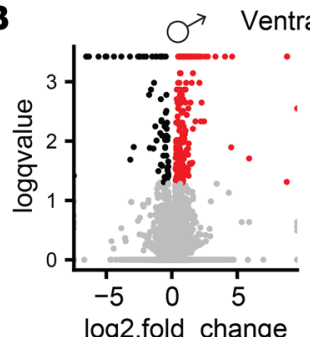

C
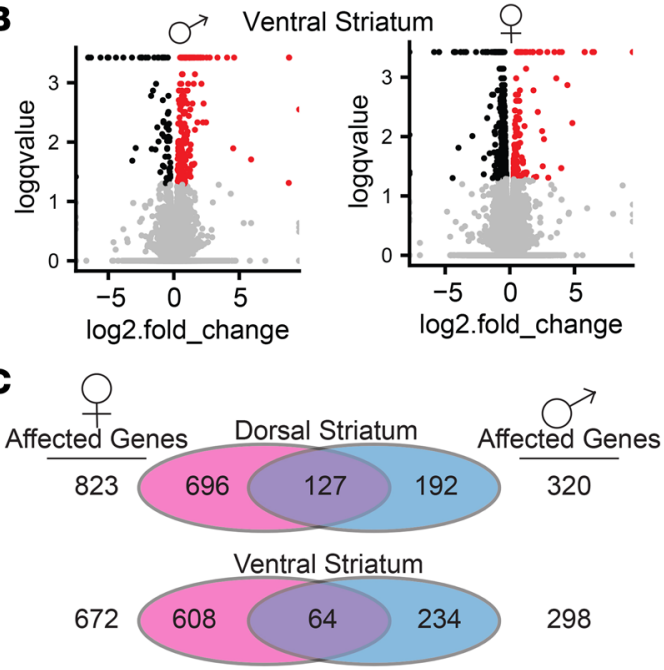

D

regulation of mut

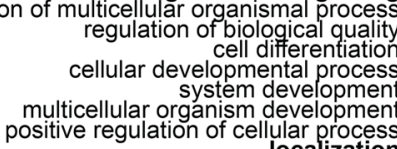
localization

$50 \begin{array}{llllllllllll}50 & 30 & 20 & 10 & 0 & 10 & 20 & 30 & 40 & 50\end{array}$ $\%$ All Sig. Affected Genes

Figure 7. RNA-sequencing of dorsal and ventral striatum reveals changes relating to signaling and neuron development. (A) Volcano plots depicting significantly regulated genes in the male and female dorsal striatum (data collected from $n=5-6$ samples per sex/diet exposure). (B) Volcano plots depicting significantly regulated genes in the male and female ventral striatum. Significance after correction for multiple testing plotted as log ${ }_{10}$ of the $\underline{Q}$ value versus fold change over CC control group. (C) Significantly affected genes in males and females and overlapping genes. (D) CO term analysis of all significantly affected genes in the dorsal striatum, with overlap between sexes plotted as a percentage of all genes in respective sex group.

between a maternal HFD and alterations to the DA system but focused primarily at the level of maternal prepregnancy obesity $(17-19,53)$. Using a combination of ex vivo and in vivo techniques, we reveal a broad range of alterations in this circuitry at multiple levels, including cell-intrinsic changes in firing and projections of midbrain DA neurons, but also affecting the excitability and wiring of postsynaptic D1 and D2 MSNs.

At the level of the midbrain DA neurons, we determined that the number of DA neurons is not affected by maternal HFD feeding during lactation, indicating that impaired neurogenesis or neuronal loss is not the causative factor for changes in DA release events and behavior. This notion is also consistent with the fact that the cell fate of dopaminergic neurons is determined during the mid to late embryonic phase in mice (reviewed in ref. 54), a period before the onset of the dietary manipulation in our model. Nevertheless, the detected changes in firing properties are consistent with the profound transcriptional changes detected in the VTA/SN of CH compared with CC mice falling in the GO term categories of regulation of ion transport and regulation of membrane potential (Figure 1). Moreover, the reduced projection formation of midbrain DA neurons coincides with the dietary manipulation and the refinement of DA projections postnatally through synaptic pruning events as well as the transcriptional changes of genes in the GO term cluster neuron development. These alterations translate into a profound reduction of func- tional DA release in DA neuron target regions. Thus, maternal HFD feeding leads to a marked reduction of stimulus-evoked DA release. It is remarkable that in light of this dramatic reduction of DA neuron activity and DA release, the observed sexually dimorphic phenotypes are relatively mild. Nevertheless, this notion is consistent with the observation that clinically relevant alterations in locomotion manifest only in Parkinson's disease, when approximately $60 \%$ of DA neurons are lost (55). Given the strong impairment of DA neuron functionality revealed in our study, it will be interesting to further investigate whether the developmental effect of altered maternal metabolism may predispose for an earlier onset of this disease.

Interestingly, despite similar changes in midbrain DA neurons identified by mRNA-sequencing, functional electrophysiologic studies, and DA neuron projection imaging, we uncovered a distinct sexual dimorphism in behavioral manifestations of changes in this circuitry. Specifically, these changes manifested in males in relation to novelty and locomotion and females with regard to reward. Recent studies in humans have shown a link between the maternal environment and sexually dimorphic-related risks of neuropsychiatric disorders in adulthood (56). Also, in rodents, sexual dimorphisms related to metabolic outcomes in offspring have been identified (reviewed in ref. 57). This further correlates with the hypothesis that similar maternal environments can have drastically different effects on the development of the offspring. Inter- 
A

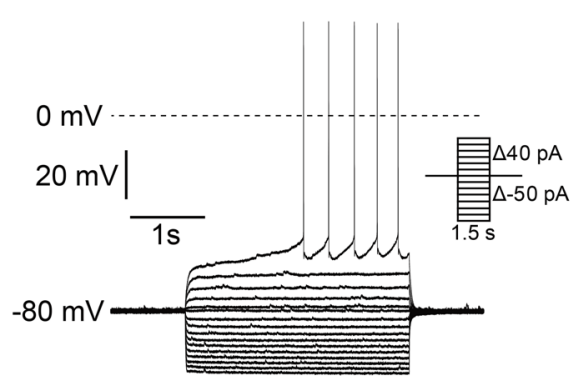

C $\quad \mathrm{CC}$

$\mathrm{CH}$

$0 \mathrm{mV}$

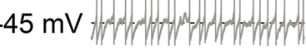

$\frac{20 \mathrm{mV}}{-90 \mathrm{mV} \quad \overline{1 \mathrm{~s}}}$

$\mathbf{F}$
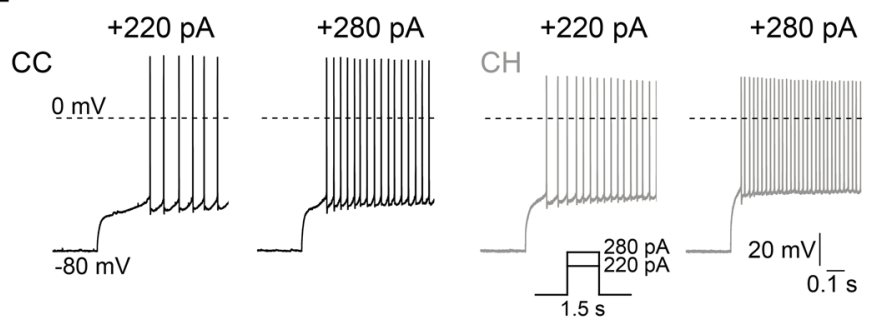

H

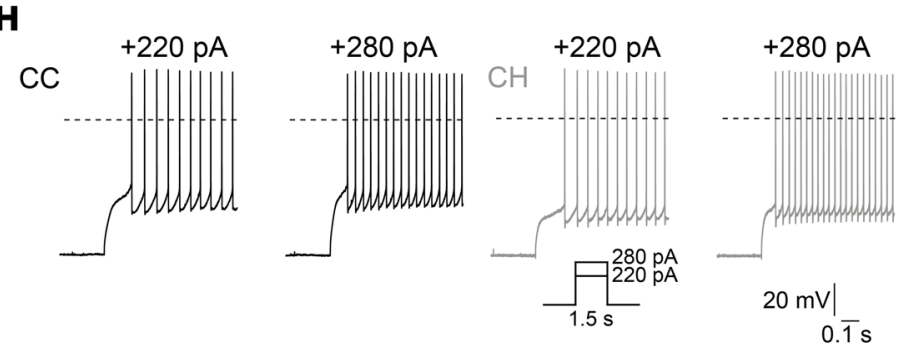

B

D

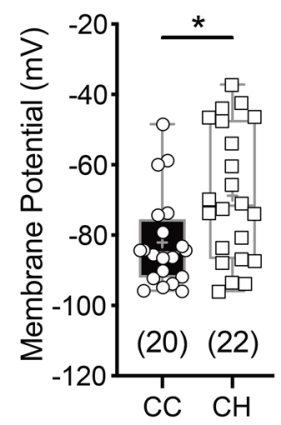

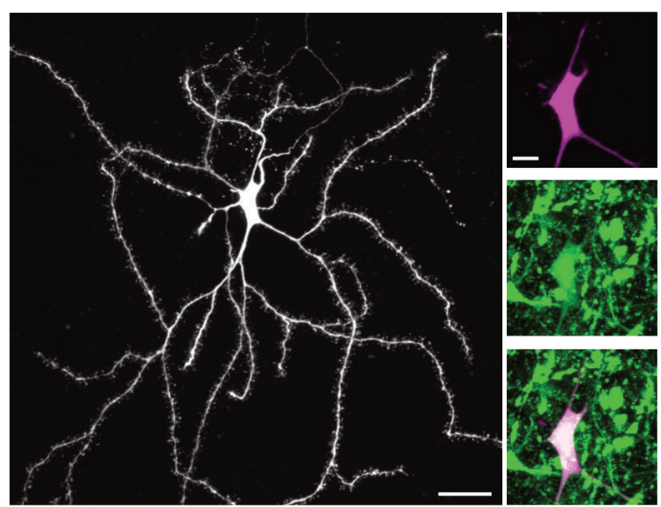

E

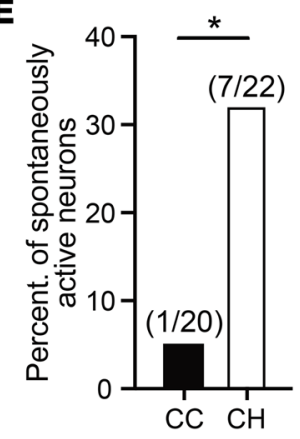

G

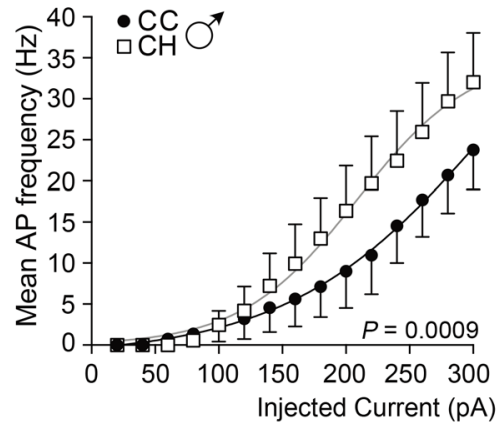

I

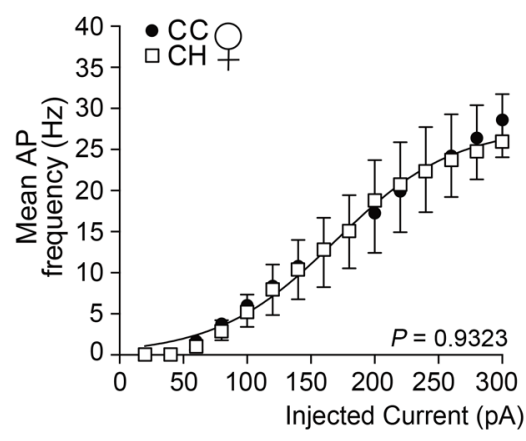

$\mathbf{L}$

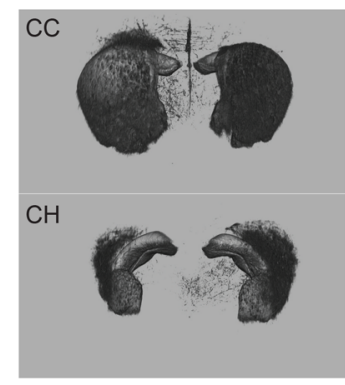

M

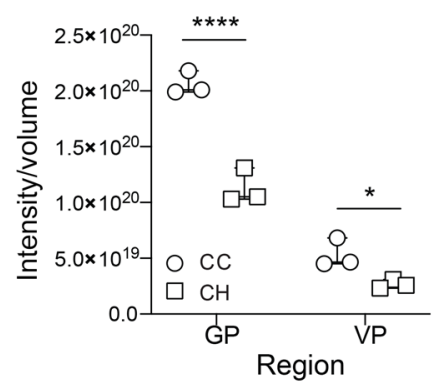


Figure 8. Electrophysiological consequences in response to maternal HFD in D1 MSNs and changes to neuron development in D2 MSNs pinpoint overall imbalance in dorsal striatum circuitry. (A) Representative electrophysiological trace and current clamp of D1 MSN. (B) Left: representative image of a D1 MSN in white. Scale bar: $50 \mu \mathrm{m}$. Right: accompanying images showing dsred labeling of TdTomato signal (top), DAT staining (middle) and the overlay of the 2 (bottom). Scale bar: $20 \mu \mathrm{m}$. (C) Recording from CC and CH D1 MSNs at baseline showing decreased membrane potential and increased spontaneous firing in CH D1 MSNs. (D) Membrane potential in CC $(n=20)$ versus CH $(n=22)$ D1 MSNs. (E) Percentage of spontaneously firing D1 MSNs. (F) Comparison of 2 different current injections in CC and CH males. (G) Quantification of mean AP frequency with increasing current injection in CC versus $\mathrm{CH}$ males. (H) Comparison of 2 different current injections in CC and CH females. (I) Quantification of mean AP frequency with increasing current injection in CC versus $\mathrm{CH}$ females. (J) Whole brain imaging in CC versus $\mathrm{CH}$ animals using the DRD1TOM. (K) Quantification of staining in J in CC $(n=4)$ versus $\mathrm{CH}(n=3)$ animals. SN, substantia nigra; Str, striatum; GPex, external globus pallidus; GPin, internal globus pallidus. (L) Whole brain imaging in CC versus $\mathrm{CH}$ animals using Adora2a ${ }^{\text {ToM }}$. (M) Quantification of staining in L in CC $(n=3)$ versus CH animals $(n=3)$. GP, globus pallidus; VP, ventral pallidum. Analysis of current injection was performed using a sigmoidal Boltzmann fit for both data sets to determine curve fit. ${ }^{*} P<0.05$, and ${ }^{* * *} P<0.001$, Fisher exact test (D), $F$ test $(\mathbf{E})$, or 2-way ANOVA with Bonferroni's post hoc analysis (K and $\mathbf{M}$ ).

estingly, with regard to neurological disease, sexual dimorphisms exist where, for example, in humans, male dominance is noted in cases of ADHD and female dominance is noted in overweight and obesity $(1,58,59)$. Of note, we have recently shown that ablation of the obesity-associated FTO gene from postsynaptic DA neurons also manifests with increased locomotion in response to novelty in male but not female mice (60). Further research is necessary to understand how the maternal metabolic environment can result in sexually dimorphic outcomes based on the broad effects on brain development and function.

Irrespective of the sex-dependent manifestation of alterations in DA-controlled behaviors as a consequence of altered maternal metabolism during lactation, it is intriguing that the behavioral changes, i.e., hyperlocomotion and increased sucrose/HFD preference, represent indicators of excessive dopaminergic action in target regions despite the robust reduction of DA neuron firing, projection, and DA release. Here, we identify a clear increase in firing and excitability of postsynaptic D1 MSNs as well as a reduction in projections of D2 MSNs in the inhibitory indirect pathway. Interestingly, in human studies of obesity risk (determined by parental body weight status), high-risk individuals displayed higher activity of striatal function (61), further supporting our finding of increased resting membrane potential of D1 MSNs. Furthermore, hyperactivation of D1 MSNs and/or suppression of D2 circuitry has been shown to be linked with autistic-like behaviors (62). Thus, in light of the reduced DA input, these changes are apparently sufficient to account for the observed behavioral effects.

In addition, we and others have shown that key metabolic circuits in the hypothalamus are affected by maternal diet as well as the influence of metabolic hormones on hypothalamic development $(9,63)$. Interestingly, these neuronal populations, namely POMC and AgRP neurons of the arcuate nucleus, send projections to the midbrain DA neurons, which express the necessary receptors for peptides released from these neurons and which have been shown to play a functional role in the adult animal (refs. 64,
65 , and reviewed in ref. 66). The function of these hypothalamic networks on dopaminergic neurons in development are not yet fully described. However, intriguing work by Dietrich et al. (67) suggests a direct interaction between hypothalamic circuits in development and the midbrain circuitry with regard to AgRP neuron ablation in the postnatal phase and resulting changes to DA-related behaviors and VTA firing properties. While we cannot directly rule out the initial influence of these known changes to key hypothalamic metabolic circuits on overall development and function of the midbrain in our model, this could provide an interesting avenue of research for understanding the relationship of hypothalamic and dopaminergic circuits in development.

In conclusion, we have unraveled the broad-based consequences of altered maternal metabolism specifically during lactation in mice on key components of the dopaminergic circuit, encompassing those affecting midbrain DA neurons and their projections as well as the downstream MSNs of the dorsal and ventral striatum. This is consistent with human studies on gestational weight gain and the manifestation of diseases associated with altered dopaminergic transmission, including obesity and neuropsychiatric disorders. Further defining the key mechanisms resulting in these changes may hold promise for developing novel therapies and interventional strategies for preventing the negative impact of maternal dietary choices on brain development and function.

\section{Methods}

Animal models. WT C57BL6/N (Charles River Labs) female mice as well as Rosa26-LSL-hM3D ${ }_{\text {Gq }}$ (68) or Rosa26-LSL-TdTomato (Jackson Laboratory, strain 007905) female mice maintained on a C57BL6/N background were placed on a control diet containing $13 \%$ calories from fat, D12450-(B) mod (ssniff Diet), at 4 weeks of age for 7 weeks, after which they were paired with WT, DAT-Cre-expressing (69), DRD1aCre-expressing (MMRRC 030778-UCD), or Adora2a-Cre-expressing (MMRRC 036158-UCD) males (also backcrossed onto a C57BL6/N background) according to the specific experiment. At birth of the pups, a subset of the mothers was switched to an HFD containing $60 \%$ calories from fat, EF D12492-(I) mod (ssniff Diet), and the remaining animals were maintained on the matched control diet, EF D12450B mod (LS ssniff Diet). Furthermore, at birth, litter sizes were adjusted, with small litters being supplemented with pups from large litters to ensure no statistical difference in litter size between groups (see Supplemental Table 5). At weaning, all offspring were maintained on the control diet for the duration of the study unless otherwise noted. For the HFD challenge in adulthood, animals were placed on HFD at 8 weeks of age. All animals were group housed in a temperature-controlled facility with a 12-hour light/12-hour dark cycle.

RNA extraction, sequencing, and analysis. Animals were sacrificed via rapid decapitation at 6 months of age and processed as described previously (64). Tissue biopsies using a $0.8-\mu \mathrm{M}$ inner diameter tissue punch (Fine Science Tools) were taken from 2-mm brain slices corresponding to the dopaminergic midbrain $(-1.0 \mathrm{~mm}$ to $+1.0 \mathrm{~mm}$ interaural $)$ or the striatum ( $+4.0 \mathrm{~mm}$ to $+6.0 \mathrm{~mm}$ interaural), as described previously (70). Bilateral micropunches were taken of the VTA, SN, and dorsal and ventral striatum and were rapidly frozen on dry ice and stored at $-80^{\circ} \mathrm{C}$ until further processing. RNA extraction was performed by first homogenizing samples in $1 \mathrm{~mL}$ Trizol LS (Life Technologies) at $3000 \mathrm{rpm}$ using 
a handheld homogenizer (IKA T 10 Basic ULTRA TURRAX disperser, Level 3). The sample was supplemented with $50 \mu \mathrm{L}$ glycogen (Ambion) and vortexed thoroughly. RNA was then extracted with the addition of $200 \mu \mathrm{L}$ chloroform (MilliporeSigma), vortexed, allowed to separate for 5 minutes at room temperature (RT), and spun at $12,200 \mathrm{~g}$ at $4^{\circ} \mathrm{C}$ for 10 minutes. The aqueous upper layer was then carefully removed, and the RNA was precipitated with equivalent amounts of cold isopropanol by inverting the tubes and placing on ice for a minimum of 15 minutes. The samples were then centrifuged at $12,200 \mathrm{~g}$ at $4^{\circ} \mathrm{C}$ for 15 minutes. The supernatant was then removed, and samples were washed once with cold $80 \%$ ethanol and centrifuged for 15 minutes at $7500 \mathrm{~g}$ at $4^{\circ} \mathrm{C}$. The supernatant was removed, and samples were allowed to air-dry for more than 5 minutes. The RNA pellet was resuspended in $30 \mu \mathrm{L}$ of Nuclease-Free water (MilliporeSigma). DNase I, DNase buffer, and SUPERase-in were added to the samples for a total volume of $37.7 \mu \mathrm{L}$ and incubated at $37^{\circ} \mathrm{C}$ for 30 minutes. The samples were then vortexed, and RNA content was measured using the Qubit RNA High Sensitivity Assay Kit (Thermo Fisher Scientific).

RNA-sequencing of samples was performed at the Cologne Center for Genomics using the Illumina TruSeq mRNA stranded protocol and the HiSeq 4000 sequencer with a PE75 read length run. Subsequent data analysis was performed as follows: the RNA-sequencing pipeline uses the GRCm38 assembly of the mouse genome and gene sets from Ensembl release 91 (71). We quantified the gene expression of each sample by (a) aligning the RNA-sequencing reads to the mm10 reference genome using hisat 2.1.0 (72) and (b) transcript assembly and quantification using the cufflinks 2.2.1 suite (73). Differentially expressed genes and isoforms between the experimental groups were calculated using cuffdiff. The data discussed in this publication were deposited in NCBI's Gene Expression Omnibus database (74) (GEO GSE147469). Overlap analysis for D1 and D2 markers used a publicly available data set (33) in comparison with the differentially expressed genes in the dorsal and ventral striatum. GO analysis was performed using the Generic Gene Ontology Term Finder (http://go.princeton.edu/cgi-bin/GOTermFinder); subsequent clustering analysis of GO Terms was performed using the REVIGO platform (http://revigo.irb.hr; ref. 75) allowing for medium similarity (0.7). Subsequent figures were generated using R.

Immunohistochemistry. Animals at 8 weeks of age were deeply anesthetized, and tissue fixation was performed via transcardial perfusion, first with sterile PBS ( $\mathrm{pH} 7.5$ ), followed by a $4 \%$ paraformaldehyde (PFA) solution in a borate buffer ( $\mathrm{pH}$ 9.5). Brains were dissected and post fixed for 4 hours in $4 \%$ PFA/borax followed by $20 \%$ sucrose in PBS at $4^{\circ} \mathrm{C}$. After more than 48 hours, brains were frozen and stored at $-80^{\circ} \mathrm{C}$ until further processing.

Brain tissue was sliced at $30 \mu \mathrm{M}$ using a freezing sledge microtome (Leica) and collected in a cryopreservant solution comprising $40 \%$ glycerol, $60 \%$ ethylene glycol, and $10 \%$ PBS stored at $-20^{\circ} \mathrm{C}$.

Samples were then processed as follows: $3 \times 10$ minutes wash in KPBS $(0.2 \mathrm{M}), 10$ minutes in $0.03 \%$ glycine, 5 minutes in KPBS $(0.2$ $\mathrm{M})$, and 10 minutes in $0.03 \%$ SDS. Samples were then incubated for 60 minutes at RT in $0.25 \%$ Triton $\mathrm{X}-100+3 \%$ normal donkey serum. Slices were then incubated overnight at $4^{\circ} \mathrm{C}$ in sheep anti-TH (1:5000, catalog AB1542, MilliporeSigma), mouse anti-calbindin (1:500, Swant, catalog C300), or rabbit anti-Aldh1a1 (1:1000, catalog ab23375, Abcam) in Signal Stain. Slices were then washed with 0.2 M KPBS and incubated for 1 hour at RT in the appropriate secondary antibody (1:500 dilution; TH experiments: donkey anti-sheep 594 [catalog A11016, Thermo Fish- er Scientific], Aldh1a1 experiments: donkey anti-rabbit-647 [catalog A31573, Thermo Fisher Scientific], Calbindin: donkey anti-mouse 488 [catalog A21202, Thermo Fisher Scientific]). All information contained within the parentheses refers to 1:400 dilutions of all secondary antibodies listed with respective primary antibody targets. After washing with $0.2 \mathrm{M}$ KPBS, slices were mounted on to Superfrost Gold Plus slides (Thermo Fisher Scientific) and coverslipped after the addition of ProLong Anti-Fade Gold Mounting Media plus DAPI (Life Technologies).

Subsequent images were acquired using a confocal Leica TCS SP-8-X microscope equipped with a $\times 20$ objective. Image analysis was performed using FIJI (NIH) by standardizing the threshold across samples and quantifying the integrated density of binarized images (SN and VTA) or by quantifying the total intensity of signal (NuAcc Core). Neuronal number quantification was also performed using FIJI.

Statistics. Immunohistochemistry data were analyzed using FIJI (Image $1.52 \mathrm{p}, \mathrm{NIH})$. OFT data were analyzed using Med Activity software (Med Associates Inc.). All statistical analysis was performed using GraphPad Prism 8 (GraphPad Software). All data are presented as one of the following: box-and-whisker plots overlaid with all individual data points and bars depicting minimum and maximum, bar graphs shown as mean \pm SEM overlaid with individual data points, and data assessing response over time. Data are shown as mean \pm SEM. Significance was defined as $P<0.05$.

Study approval. All animal experiments were performed in accordance with protocols approved by the local governmental authorities (Bezirksregierung Köln). Voltammetry experiments were conducted under the Animal (Scientific Procedures) Act 1986, United Kingdom, and the Local Ethical Review Committee at the University of Oxford. Voltammetry experiments were approved by the UK Home Office as well as the Oxford Animal Welfare and Ethical Review Board.

\section{Author contributions}

RNL and JCB designed the experiments. RNL performed the maternal diet interventions and offspring tracking, behavioral tests, imaging experiments, and analyses. RNL, LMB, MEW, and JCB designed and LMB performed the FSCV experiments. TJP provided the code to analyze the FSCV experiments. JCB, RNL, $\mathrm{SH}$, and $\mathrm{P}$ Kloppenburg designed and $\mathrm{SH}$ performed the electrophysiology experiments. P Klemm analyzed the RNA-sequencing data. RNL and SH created the figures. RNL and JCB drafted the manuscript with input from all authors.

\section{Acknowledgments}

We would like to thank Nils Goran-Larsson (Max Planck Institute for the Biology of Ageing, Cologne) for providing the DatCre animals. In addition, we would like to thank Svenja Corneliussen for her technical help. LMB was supported by a National Science Foundation Postdoctoral Research Fellowship in Biology (NSF grant number 1811713). MEW was supported by a Wellcome Senior Research Fellowship (202831/Z/16/Z). JCB received funding within the Excellence Initiative by German federal and state governments (CECAD), from the DZD, and from the European Union through the ERC advanced grant 742106 "SYNEME."

Address correspondence to: Jens C. Brüning, Max Planck Institute for Metabolism Research, Gleueler Street 50, 50931 Cologne, Germany. Phone: 49.221.4726.202; Email: bruening@sf.mpg.de. 
1. GBD 2015 Obesity Collaborators, et al. Health effects of overweight and obesity in 195 countries over 25 years. $N$ Engl J Med. 2017;377(1):13-27.

2. Andersen CH, Thomsen PH, Nohr EA, Lemcke S. Maternal body mass index before pregnancy as a risk factor for ADHD and autism in children. Eur Child Adolesc Psychiatry. 2018;27(2):139-148.

3. Kong L, Norstedt G, Schalling M, Gissler M, Lavebratt $\mathrm{C}$. The risk of offspring psychiatric disorders in the setting of maternal obesity and diabetes. Pediatrics. 2018;142(3):e20180776.

4. Rivera HM, Christiansen KJ, Sullivan EL. The role of maternal obesity in the risk of neuropsychiatric disorders. Front Neurosci. 2015;9:194.

5. Godfrey KM, et al. Influence of maternal obesity on the long-term health of offspring. Lancet Diabetes Endocrinol. 2017;5(1):53-64.

6. Black RE, et al. Maternal and child undernutrition and overweight in low-income and middle-income countries. Lancet. 2013;382(9890):427-451.

7. Goldstein RF, et al. Association of gestational weight gain with maternal and infant outcomes: a systematic review and meta-analysis. JAMA. 2017;317(21):2207-2225

8. Lindsay KL, Buss C, Wadhwa PD, Entringer S. The interplay between nutrition and stress in pregnancy: implications for fetal programming of brain development. Biol Psychiatry. 2019;85(2):135-149.

9. Vogt MC, et al. Neonatal insulin action impairs hypothalamic neurocircuit formation in response to maternal high-fat feeding. Cell. 2014;156(3):495-509.

10. Gali Ramamoorthy T, et al. Maternal overnutrition programs epigenetic changes in the regulatory regions of hypothalamic Pomc in the offspring of rats. Int JObes (Lond). 2018;42(8):1431-1444.

11. Dearden L, Ozanne SE. Early life origins of metabolic disease: Developmental programming of hypothalamic pathways controlling energy homeostasis. Front Neuroendocrinol. 2015;39:3-16.

12. van der Klaauw AA, et al. Human semaphorin 3 variants link melanocortin circuit development and energy balance. Cell. 2019;176(4):729-742.e18.

13. Rodriguez A, et al. Maternal adiposity prior to pregnancy is associated with ADHD symptoms in offspring: evidence from three prospective pregnancy cohorts. Int JObes (Lond). 2008;32(3):550-557.

14. Gage SH, Lawlor DA, Tilling K, Fraser A. Associations of maternal weight gain in pregnancy with offspring cognition in childhood and adolescence: findings from the Avon Longitudinal Study of Parents and Children. Am JEpidemiol. 2013;177(5):402-410.

15. Keim SA, Pruitt NT. Gestational weight gain and child cognitive development. Int J Epidemiol. 2012;41(2):414-422.

16. Naef L, Srivastava L, Gratton A, Hendrickson $\mathrm{H}$, Owens SM, Walker CD. Maternal high fat diet during the perinatal period alters mesocorticolimbic dopamine in the adult rat offspring: reduction in the behavioral responses to repeated amphetamine administration. Psychopharmacology (Berl). 2008;197(1):83-94.

17. Vucetic Z, Kimmel J, Totoki K, Hollenbeck E, Reyes TM. Maternal high-fat diet alters methylation and gene expression of dopamine and opioid-related genes. Endocrinology. 2010;151(10):4756-4764.

18. Peleg-Raibstein D, et al. Enhanced sensitivity to drugs of abuse and palatable foods following maternal overnutrition. Transl Psychiatry. 2016;6(10):e911.

19. Sarker G, et al. Transgenerational transmission of hedonic behaviors and metabolic phenotypes induced by maternal overnutrition. Transl Psychiatry. 2018;8(1):195.

20. Semple BD, Blomgren K, Gimlin K, Ferriero DM, Noble-Haeusslein LJ. Brain development in rodents and humans: Identifying benchmarks of maturation and vulnerability to injury across species. Prog Neurobiol. 2013;106-107:1-16.

21. Knuesel I, et al. Maternal immune activation and abnormal brain development across CNS disorders. Nat Rev Neurol. 2014;10(11):643-660.

22. Sharon G, Sampson TR, Geschwind DH, Mazmanian SK. The central nervous system and the gut microbiome. Cell. 2016;167(4):915-932.

23. Rice D, Barone S. Critical periods of vulnerability for the developing nervous system: evidence from humans and animal models. Environ Health Perspect. 2000;108 Suppl 3:511-533.

24. Workman AD, Charvet CJ, Clancy B, Darlington RB, Finlay BL. Modeling transformations of neurodevelopmental sequences across mammalian species. J Neurosci. 2013;33(17):7368-7383.

25. Mendes-da-Silva C, Giriko CÁ, Mennitti LV, Hosoume LF, Souto Tdos S, Silva AV. Maternal high-fat diet during pregnancy or lactation changes the somatic and neurological development of the offspring. Arq Neuropsiquiatr. 2014;72(2):136-144.

26. Ungless MA, Grace AA. Are you or aren't you? Challenges associated with physiologically identifying dopamine neurons. Trends Neurosci. 2012;35(7):422-430.

27. Neuhoff H, Neu A, Liss B, Roeper J. I(h) channels contribute to the different functional properties of identified dopaminergic subpopulations in the midbrain. J Neurosci. 2002;22(4):1290-1302.

28. Lacey MG, Mercuri NB, North RA. Two cell types in rat substantia nigra zona compacta distinguished by membrane properties and the actions of dopamine and opioids. JNeurosci. 1989;9(4):1233-1241.

29. Richards CD, Shiroyama T, Kitai ST. Electrophysiological and immunocytochemical characterization of GABA and dopamine neurons in the substantia nigra of the rat. Neuroscience. 1997;80(2):545-557.

30. Lippert RN, et al. Time-dependent assessment of stimulus-evoked regional dopamine release. Nat Commun. 2019;10(1):336

31. Breton JM, et al. Relative contributions and mapping of ventral tegmental area dopamine and GABA neurons by projection target in the rat. JComp Neurol. 2019;527(5):916-941.

32. Roberts JG, Toups JV, Eyualem E, McCarty GS, Sombers LA. In situ electrode calibration strategy for voltammetric measurements in vivo. Anal Chem. 2013;85(23):11568-11575.

33. Heiman M, et al. A translational profiling approach for the molecular characterization of CNS cell types. Cell. 2008;135(4):738-748.

34. Alcacer C, Andreoli L, Sebastianutto I, Jakobsson J, Fieblinger T, Cenci MA. Chemogenetic stimulation of striatal projection neurons modulates responses to Parkinson's disease therapy. JClin Invest. 2017;127(2):720-734.
35. Chartoff EH, Marck BT, Matsumoto AM, Dorsa $\mathrm{DM}$, Palmiter RD. Induction of stereotypy in dopamine-deficient mice requires striatal D1 receptor activation. Proc Natl Acad Sci U S A. 2001;98(18):10451-10456.

36. Zhu X, Ottenheimer D, DiLeone RJ. Activity of D1/2 receptor expressing neurons in the nucleus accumbens regulates running, locomotion, and food intake. Front Behav Neurosci. 2016;10:66.

37. Hegarty SV, Sullivan AM, O'Keeffe GW. Midbrain dopaminergic neurons: a review of the molecular circuitry that regulates their development. Dev Biol. 2013;379(2):123-138.

38. Tau GZ, Peterson BS. Normal development of brain circuits. Neuropsychopharmacology. 2010;35(1):147-168

39. Kuo HY, Liu FC. Synaptic wiring of corticostriatal circuits in basal ganglia: insights into the pathogenesis of neuropsychiatric disorders. eNeuro. 2019;6(3):ENEURO.0076-19.2019.

40. Gallardo CM, et al. Dopamine receptor 1 neurons in the dorsal striatum regulate food anticipatory circadian activity rhythms in mice. Elife. 2014;3:e03781.

41. Kenny PJ. Reward mechanisms in obesity: new insights and future directions. Neuron. 2011;69(4):664-679.

42. Palmiter RD. Dopamine signaling in the dorsa striatum is essential for motivated behaviors: lessons from dopamine-deficient mice. Ann $N Y$ Acad Sci. 2008;1129:35-46.

43. Johnson PM, Kenny PJ. Dopamine D2 receptors in addiction-like reward dysfunction and compulsive eating in obese rats. Nat Neurosci. 2010;13(5):635-641.

44. Volkow ND, Wang GJ, Baler RD. Reward, dopamine and the control of food intake: implications for obesity. Trends Cogn Sci (Regul Ed). 2011;15(1):37-46.

45. Volkow ND, Wang GJ, Fowler JS, Telang F. Overlapping neuronal circuits in addiction and obesity: evidence of systems pathology. Philos Trans $R$ Soc Lond, B, Biol Sci. 2008;363(1507):3191-3200.

46. Volkow ND, Wang GJ, Tomasi D, Baler RD. Obesity and addiction: neurobiological overlaps. Obes Rev. 2013;14(1):2-18.

47. Wang GJ, et al. Brain dopamine and obesity. Lancet. 2001;357(9253):354-357.

48. Stice E, Spoor S, Bohon C, Small DM. Relation between obesity and blunted striatal response to food is moderated by TaqIA A1 allele. Science. 2008;322(5900):449-452.

49. Fernandes MF, et al. Leptin suppresses the rewarding effects of running via STAT3 signaling in dopamine neurons. Cell Metab. 2015;22(4):741-749.

50. Fulton S, et al. Leptin regulation of the mesoaccumbens dopamine pathway. Neuron 2006;51(6):811-822.

51. Fulton S, Woodside B, Shizgal P. Modulation of brain reward circuitry by leptin. Science. 2000;287(5450):125-128.

52. Hommel JD, et al. Leptin receptor signaling in midbrain dopamine neurons regulates feeding. Neuron. 2006;51(6):801-810.

53. Rivera HM, et al. Maternal high-fat diet and obesity impact palatable food intake and dopamine signaling in nonhuman primate offspring. Obesity 
(Silver Spring). 2015;23(11):2157-2164.

54. La Manno G, et al. Molecular diversity of midbrain development in mouse, human, and stem cells. Cell. 2016;167(2):566-580.e19.

55. Dauer W, Przedborski S. Parkinson's disease: mechanisms and models. Neuron. 2003;39(6):889-909.

56. Ursini G, et al. Convergence of placenta biology and genetic risk for schizophrenia. Nat Med. 2018;24(6):792-801.

57. Dearden L, Bouret SG, Ozanne SE. Sex and gender differences in developmental programming of metabolism. Mol Metab. 2018;15:8-19.

58. Hales CM, Fryar CD, Carroll MD, Freedman DS, Ogden CL. Trends in obesity and severe obesity prevalence in US youth and adults by sex and age, 2007-2008 to 2015-2016. JAMA. 2018;319(16):1723-1725.

59. Nøvik TS, et al. Influence of gender on attention-deficit/hyperactivity disorder in Europe--ADORE. Eur Child Adolesc Psychiatry. 2006;15(suppl 1):I15-I24.

60. Ruud J, et al. The fat mass and obesity-associated protein (FTO) regulates locomotor responses to novelty via D2R medium spiny neurons. Cell Rep. 2019;27(11):3182-3198.e9.

61. Stice E, Yokum S, Burger KS, Epstein LH, Small
DM. Youth at risk for obesity show greater activation of striatal and somatosensory regions to food. JNeurosci. 2011;31(12):4360-4366.

62. Lee Y, et al. Excessive D1 dopamine receptor activation in the dorsal striatum promotes autistic-like behaviors. Mol Neurobiol. 2018;55(7):5658-5671.

63. Bouret SG, Draper SJ, Simerly RB. Trophic action of leptin on hypothalamic neurons that regulate feeding. Science. 2004;304(5667):108-110.

64. Lippert RN, Ellacott KL, Cone RD. Gender-specific roles for the melanocortin-3 receptor in the regulation of the mesolimbic dopamine system in mice. Endocrinology. 2014;155(5):1718-1727.

65. Pandit R, et al. Melanocortin 3 Receptor signaling in midbrain dopamine neurons increases the motivation for food reward. Neuropsychopharmacology. 2016;41(9):2241-2251.

66. Roseberry AG, Stuhrman K, Dunigan AI. Regulation of the mesocorticolimbic and mesostriatal dopamine systems by $\alpha$-melanocyte stimulating hormone and agouti-related protein. Neurosci Biobehav Rev. 2015;56:15-25.

67. Dietrich MO, et al. AgRP neurons regulate development of dopamine neuronal plasticity and nonfood-associated behaviors. Nat Neurosci. 2012;15(8):1108-1110.
68. Koehler S, et al. Single and transient $\mathrm{Ca}^{2+}$ peaks in podocytes do not induce changes in glomerular filtration and perfusion. Sci Rep. 2016;6:35400.

69. Ekstrand MI, et al. Progressive parkinsonism in mice with respiratory-chain-deficient dopamine neurons. Proc Natl Acad Sci U S A. 2007;104(4):1325-1330.

70. Paxinos G, Franklin K. The Mouse Brain in Stereotaxic Coordinates. 2nd ed. Oxford, United Kingdom: Elsevier Academic Press; 2004.

71. Yates A, et al. Ensembl 2016. Nucleic Acids Res. 2016;44(D1):D710-D716.

72. Kim D, Langmead B, Salzberg SL. HISAT: a fast spliced aligner with low memory requirements. Nat Methods. 2015;12(4):357360.

73. Trapnell C, et al. Transcript assembly and quantification by RNA-Seq reveals unannotated transcripts and isoform switching during cell differentiation. Nat Biotechnol. 2010;28(5):511515.

74. Edgar R, Domrachev M, Lash AE. Gene Expression Omnibus: NCBI gene expression and hybridization array data repository. Nucleic Acids Res. 2002;30(1):207-210.

75. Supek F, Bošnjak M, Škunca N, Šmuc T. REVIGO summarizes and visualizes long lists of gene ontology terms. PLoS One. 2011;6(7):e21800. 